\title{
Neutron and Gamma Ray Pulse Shape Discrimination with Polyvinyltoluene
}

\author{
Azaree Lintereur \\ James Ely \\ Jean Stave \\ Benjamin Mcdonald
}

March 2012

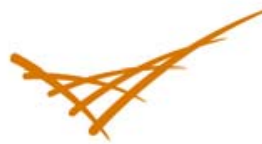

Pacific Northwest

NATIONAL LABORATORY

Proudly Operated by Battelle Since 1965 


\title{
DISCLAIMER
}

This report was prepared as an account of work sponsored by an agency of the United States Government. Neither the United States Government nor any agency thereof, nor Battelle Memorial Institute, nor any of their employees, makes any warranty, express or implied, or assumes any legal liability or responsibility for the accuracy, completeness, or usefulness of any information, apparatus, product, or process disclosed, or represents that its use would not infringe privately owned rights. Reference herein to any specific commercial product, process, or service by trade name, trademark, manufacturer, or otherwise does not necessarily constitute or imply its endorsement, recommendation, or favoring by the United States Government or any agency thereof, or Battelle Memorial Institute. The views and opinions of authors expressed herein do not necessarily state or reflect those of the United States Government or any agency thereof.

\author{
PACIFIC NORTHWEST NATIONAL LABORATORY \\ operated by \\ BATTELLE \\ for the \\ UNITED STATES DEPARTMENT OF ENERGY \\ under Contract DE-AC05-76RL01830
}

Printed in the United States of America

\section{Available to DOE and DOE contractors from the \\ Office of Scientific and Technical Information, \\ P.O. Box 62, Oak Ridge, TN 37831-0062; \\ ph: (865) 576-8401 \\ fax: (865) 576-5728 \\ email: reports@adonis.osti.gov}

Available to the public from the National Technical Information Service, U.S. Department of Commerce, 5285 Port Royal Rd., Springfield, VA 22161

ph: (800) 553-6847

fax: (703) 605-6900

email: orders@ntis.fedworld.gov

online ordering: http://www.ntis.gov/ordering.htm 


\section{Neutron and Gamma Ray Pulse Shape Discrimination with Polyvinyltoluene}

Azaree Lintereur

James Ely

Jean Stave

Benjamin Mcdonald

March 2012

Pacific Northwest National Laboratory

Richland, Washington 99352 


\section{Executive Summary}

The goal of this research effort was to test the ability of two polyvinyltoluene research samples to produce recordable, distinguishable signals in response to gamma rays and neutrons. Pulse shape discrimination was performed to identify if the signal was generated by a gamma ray or a neutron. A standard figure of merit for pulse shape discrimination was used to quantify the gamma ray - neutron pulse separation. Measurements were made with gamma ray and neutron sources with and without shielding. The best figure of merit obtained was 1.77; this figure of merit was achieved with the first sample in response to an un-moderated ${ }^{252} \mathrm{Cf}$ source shielded with $5.08 \mathrm{~cm}$ of lead. 


\section{Acronyms and Abbreviations}

$\varepsilon$

FOM

FWHM

LLNL

PMT

PMCA

PNNL

PSA

PSD

PVT

Qratio detection efficiency

figure-of-merit

full-width at half-maximum

Lawrence Livermore National Laboratory

pocket multichannel analyzer

photomultiplier tube

Pacific Northwest National Laboratory

pulse shape analyzer

pulse shape discrimination

polyvinyltoluene

ratio of the charge in the tail of the pulse to the total charge in the pulse 


\section{Contents}

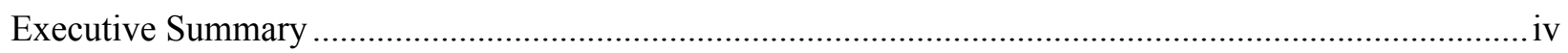

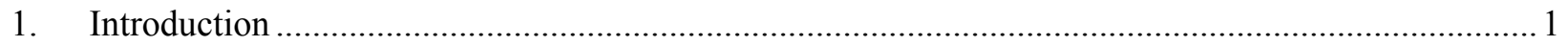

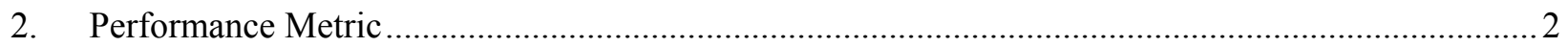

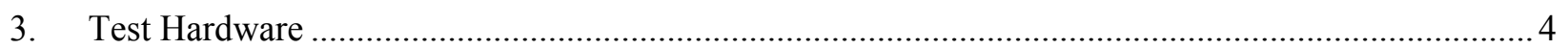

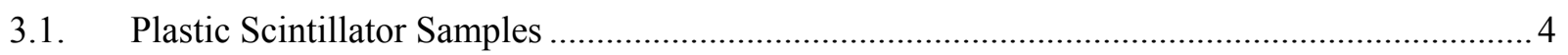

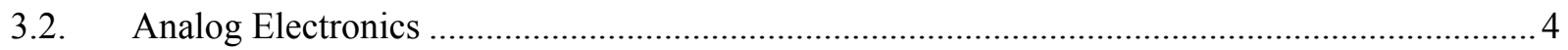

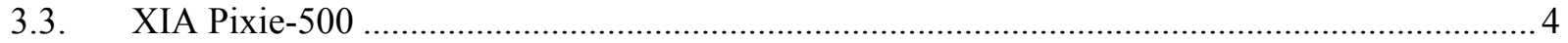

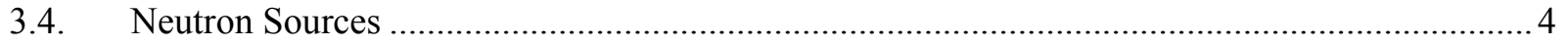

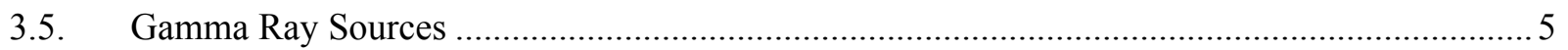

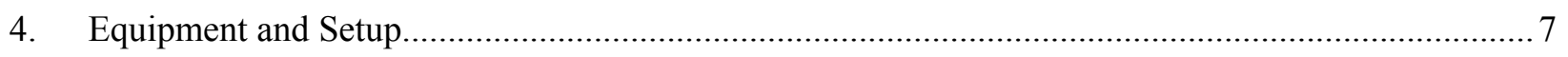

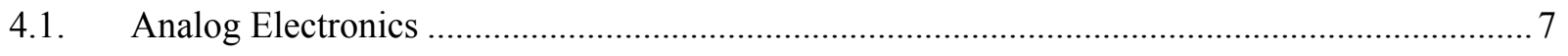

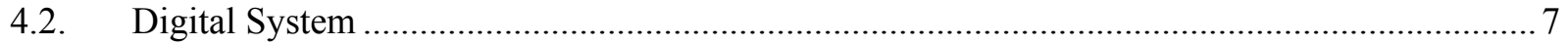

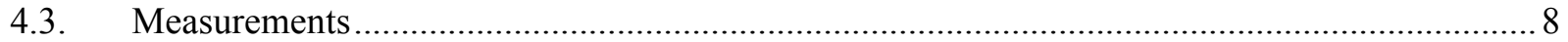

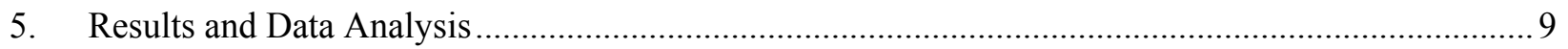

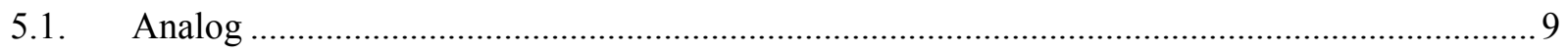

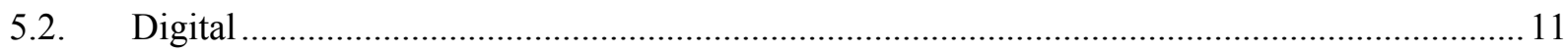

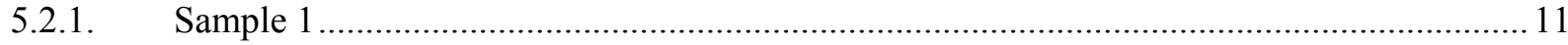

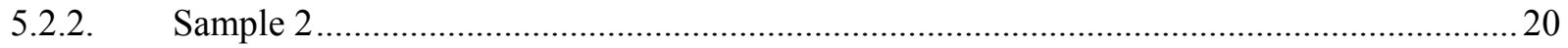

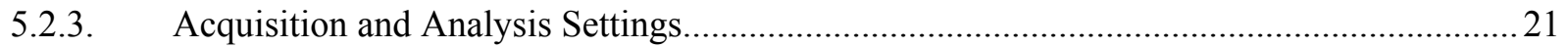

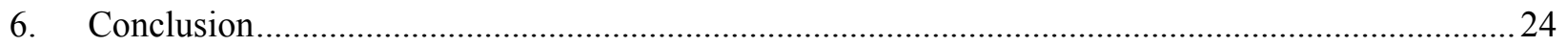

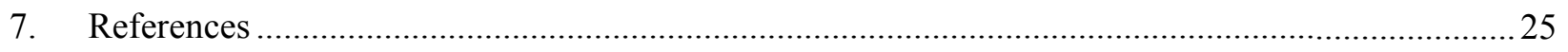

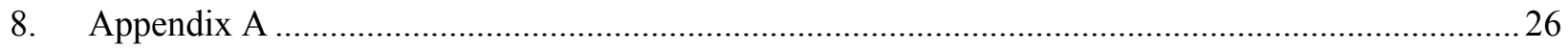

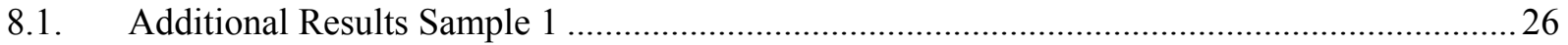

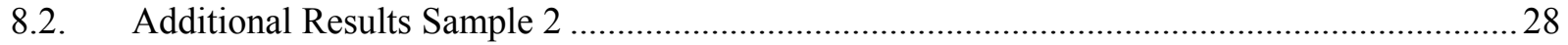




\section{Figures and Tables}

\section{Figures}

Figure 1: Definition of the FOM for gamma ray-neutron separation based on PSD (adapted from Figure 1, "Pulse Shape Discrimination in Impure and Mixed Single-Crystal Organic Scintillators," N. Zaitseva et al., 2011, IEEE Transactions on Nuclear Science, 58:6, p. 3411).

Figure 2: Digital experimental setup with a bare source and no shielding (a) and with a lead brick and

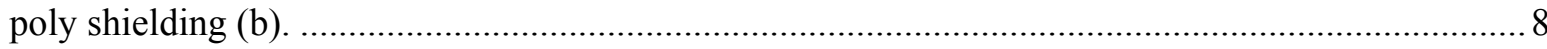

Figure 3: Analog pulse shape data from the PVT sample with a ${ }^{252} \mathrm{Cf}$ source and a ${ }^{137} \mathrm{Cs}$ source. The results are shown on a log scale (a) to show the complete set of data (note that the channels with zero counts could not be shown on the log plot) and zoomed in on a linear scale (b) which was used

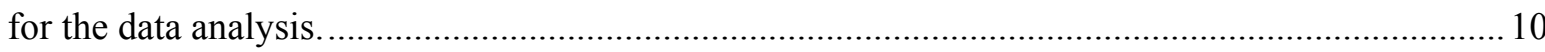

Figure 4: Individual gamma ray (a) and neutron (b) pulses recorded with Sample 1 in response to a ${ }^{252} \mathrm{Cf}$ source. The same pulses are shown on a log scale (gamma ray (c) and neutron (d)) to illustrate the different tail shape.

Figure 5: The ratio of the charge in the tail of the pulses to the total charge associated with the pulse versus peak height obtained from Sample 1 with a bare ${ }^{252} \mathrm{Cf}$ source shielded by $5.08 \mathrm{~cm}$ of $\mathrm{Pb}$. Note that a "neutron region" can be identified for peak heights greater than 0.5 and with Qratios above approximately 0.25 but there is not a clear gamma ray-neutron distinction.

Figure 6: Histogram of the Qratio data shown in Figure 5. Note the lack of clear gamma ray and neutron peaks.

Figure 7: Ten over-laid traces recorded in response to a ${ }^{252} \mathrm{Cf}$ source with Sample 1 ............................ 14

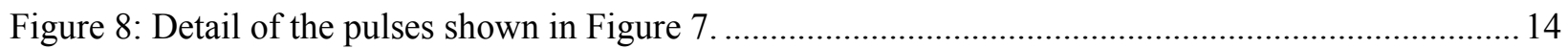

Figure 9: Ten over-laid pulses recorded in response to a ${ }^{252} \mathrm{C}$ f source by Sample 1 with the maximum

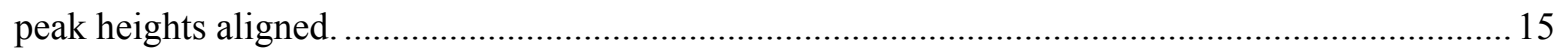

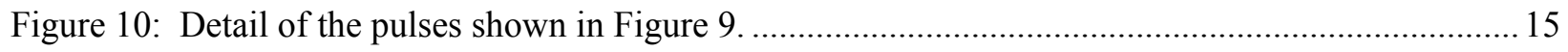

Figure 11: Ratio of the charge in the tail of the pulse to the total charge in the pulse as a function of the peak height, after the pulse peak height maximums were aligned. Note the clear neutron (higher) and gamma ray (lower) regions.

Figure 12: Histogram of the data in Figure 11. Note the clear gamma ray (lower Qratio) and neutron (higher Qratio) peaks.

Figure 13: The same data plotted in Figure 11 with an upper and lower threshold (1.4 and 0.25 respectively) applied to the Qratio.

Figure 14: Histogram of the data in Figure 13.

Figure 15: The Qratio as a function of peak height for the pulses recorded by Sample 1 in response to a ${ }^{137} \mathrm{Cs}$ source with the pulse peaks aligned and an upper and lower level threshold (1.4 and 0.25

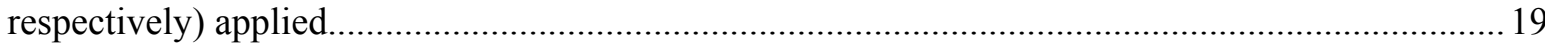

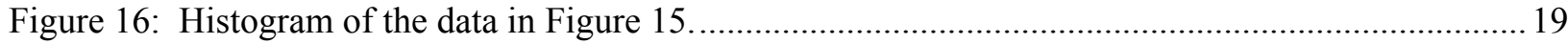


Figure 17: Qratio as a function of peak height for the aligned pulses recorded in response to ${ }^{252} \mathrm{Cf}$ by

Sample 2 with an upper (1.4) and lower (0.25) threshold applied to the Qratio. 20

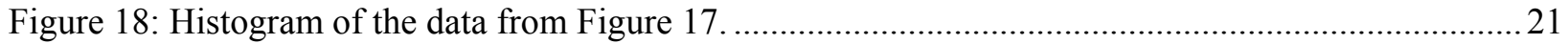

Figure 19: Lower level threshold, total number of counts, and FOM relationship. The red dotted line represents the commonly accepted minimum FOM required for adequate neutron-gamma ray separation.

Figure 20: Results from the pulses recorded by Sample 1 in response to a bare ${ }^{252} \mathrm{Cf}$ source with the pulse peaks aligned and an upper (1.4) and lower (0.25) threshold applied........................................26

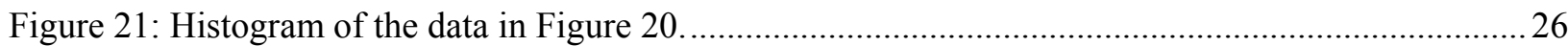

Figure 22: Results from the pulses recorded by Sample 1 in response to a bare AmBe source shielded by $5.08 \mathrm{~cm}$ of $\mathrm{Pb}$ with the pulse peaks aligned and an upper (1.4) and lower (0.25) threshold applied. 27

Figure 23: Histogram of the data shown in Figure 22.

Figure 24: Results from the pulses recorded by Sample 2 in response to a bare AmBe source shielded by $5.08 \mathrm{~cm}$ of $\mathrm{Pb}$ with the pulse peaks aligned and an upper (1.4) and lower (0.25) threshold applied. 28

Figure 25: Histogram of the data shown in Figure 24. 28

\section{Tables}

Table 1: Neutron sources used for this measurement campaign........................................................ 5

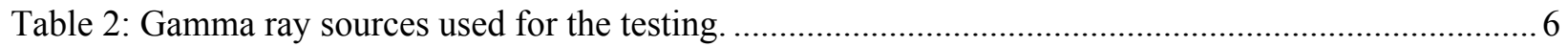

Table 3: PIXIE-500 settings used for data acquisition with the PVT sample............................................ 7

Table 4: The event rates recorded with different threshold settings during 1 minute acquisitions with Sample 2 in response to the ${ }^{252} \mathrm{Cf}$ source shielded with $5.08-\mathrm{cm}$ of $\mathrm{Pb}$.

Table 5: The affect of the windows used to define the pulse peak and the total pulse on the FOM calculated for each sample. 


\section{Introduction}

Organic scintillators produce light by both prompt and delayed fluorescence. The prompt decay time is typically a couple of nanoseconds, while the delayed decay time is normally on the order of hundreds of nanoseconds. The majority of the light is produced by the prompt decay; however, the amount of light in the delayed component often varies as a function of the type of particle causing the excitation (Knoll 2000). The variation in the amount of light produced by delayed fluorescence can be utilized to distinguish different types of particles; this technique is known as pulse shape discrimination (PSD).

Neutron interactions in organic scintillators produce scattered protons through elastic scattering; protons have a short range and generate a high concentration of triplet states, which decay by delayed fluorescence. By contrast, gamma ray interactions in organic scintillators produce scattered electrons. Electrons have a longer range than protons and generate a lower concentration of triplet states (electrons are more likely to produce excited singlet states, which decay by prompt fluorescence) (Zaitseva 2011a). The difference in the pulse shape of the signal as a result of the ratio of prompt to delayed fluorescence produced by different types of radiation makes PSD a popular method of high-energy neutron detection in an environment where gamma rays are present.

Polyvinyltoluene (PVT), which has a similar composition to liquid scintillators (often used for PSD) has not traditionally been used for PSD due to an inability to produce distinguishable signals between fast neutrons and gamma rays. Pulse shape discrimination has been performed with plastics other than PVT, but the results have been inferior to what can be achieved with liquid scintillators (Winyard 1971); the lack of a distinct neutron signal has led to the convention that plastic scintillators are not appropriate for neutron detection. Plastic scintillators have attributes that make them more attractive than liquid scintillators for some applications (such as no risk of leaks). There have been attempts to fabricate plastics specifically for use in situations where gamma ray-neutron discrimination is required, but clear separation, using PSD, between gamma rays and neutrons was not achieved (Hamel 2008). However, it has recently been discovered that increasing the fluorescent dye in organic crystals can improve PSD capabilities (Zaitseva 2011a), and the same techniques were applied to PVT to generate a neutron sensitive plastic scintillator (Zaitseva 2012).

Two PVT research samples (2.54-cm diameter) were fabricated and provided by Lawrence Livermore National Laboratory (LLNL) to Pacific Northwest National Laboratory (PNNL) for testing. The response of the samples to neutrons and gamma rays was measured, and the results are reported here. The purpose of this study was to verify that gamma ray-neutron separation could be achieved with the research samples using data collected at (and the post processing methods employed by) PNNL. The results presented were not used to validate any measurements made by LLNL, as the exact concentrations of the fluorescent dye in the samples were not reported, nor were identical post-processing methods employed. 


\section{Performance Metric}

A fast neutron detector that is appropriate for use in situations where gamma rays are present is one that can produce clearly separated neutron and gamma ray signals. The separation between the neutron and gamma ray signals can be quantified and used to determine a performance metric. A standard figure of merit (FOM) has been identified for fast neutron detectors and is used to establish their ability to discriminate between pulses generated by gamma rays and pulses generated by neutrons. The FOM is calculated after PSD has been performed to identify the neutron and gamma ray pulses.

The PSD techniques used to distinguish between the pulses from neutrons and the pulses from gamma rays rely on the differences in the pulse shapes produced. The pulses generated by neutrons will have a longer tail than the pulses generated by gamma rays, as the neutron pulses are the result of triplet state interactions (delayed fluorescence) and the pulses produced by gamma rays are the result of singlet state de-excitation (prompt fluorescence). Thus, the difference in the ratio of the charge in the tail of the pulse to the total charge in the pulse (the Qratio) can be calculated and used to discern which type of radiation generated the pulse. The Qratio for neutron pulses should be larger than the Qratio for gamma ray pulses for the same total charge deposited (Figure 1). The FOM is calculated from the histogram of the Qratio versus peak height data. The FOM is defined as (note that this definition assumes that the pulse distributions are Gaussian):

$$
F O M=\frac{S}{\delta_{\text {neutron }}+\delta_{\text {gamma }}} \quad \text { Equation } 1
$$

$S \equiv$ the distance between the gamma ray and neutron peaks

$\delta \equiv$ the full width at half maximum (FWHM) of the peaks.

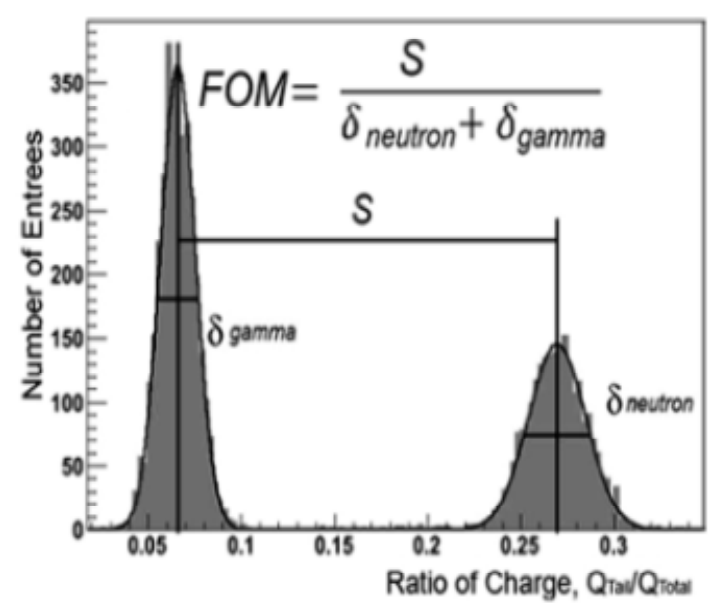

Figure 1: Definition of the FOM for gamma ray-neutron separation based on PSD (adapted from Figure 1, "Pulse Shape Discrimination in Impure and Mixed Single-Crystal Organic Scintillators," N. Zaitseva et al., 2011, IEEE Transactions on Nuclear Science, 58:6, p. 3411). 
The definition of the FOM illustrates that the larger the FOM the better the performance of the detector for gamma ray discrimination. A baseline performance requirement can be established by starting with the definition that for two peaks to be considered well separated $\mathrm{S}>3\left(\sigma_{\mathrm{gamma}}+\right.$ $\left.\sigma_{\text {neutron}}\right)$, where $\sigma$ is the standard deviation. For a Gaussian distribution the FWHM $=2.36 \sigma$. Substituting these definitions into the equation 1 yields the result:

$$
F O M \geq \frac{3\left(\sigma_{\text {neutron }}+\sigma_{\text {gamma }}\right)}{2.36\left(\sigma_{\text {neutron }}+\sigma_{\text {gamma }}\right)}=1.27 \quad \text { Equation } 2
$$

Thus, any detector with a FOM above 1.27 can be considered to have adequate PSD for fast neutron detection in the presence of gamma rays (Zaitseva 2012). 


\section{Test Hardware}

\subsection{Plastic Scintillator Samples}

Two plastic scintillating right circular cylindrical samples 1.80 - $\mathrm{cm}$ thick with diameters of 2.54$\mathrm{cm}$ were obtained from LLNL for these measurements. The samples were polyvinyltoluene based plastic scintillators loaded with fluorescent compounds (the primary and secondary dyes were 2,5-diphenyloxazole and 9,10-diphenylanthracene, respectively). The ratios of the primary and secondary fluorescent dyes were chosen to produce a plastic sensitive to both gamma rays and neutrons with a high light yield (Zaitseva 2012). The same measurements were made with both samples to verify the results.

\subsection{Analog Electronics}

Analog electronics were used to collect the initial data. The sample was coupled with optical grease to a 2.54-cm diameter Hamamatsu R1924A photomultiplier tube (PMT), and the PMT was connected to a Canberra 2005 preamplifier. The signal was routed from the preamplifier into a Canberra 2024 amplifier module, and then into an Ortec 458 pulse shape analyzer (PSA). The output of the PSA was connected to an Amptek MCA-8000A Pocket Multichannel Analyzer (PMCA), which binned the data according to pulse height and then transmitted the data to a computer.

\subsection{XIA Pixie-500}

Data was also collected with digital electronics. Digitized pulse data was acquired using a 12bit, $500-\mathrm{MHz}$ version of the DGF Pixie-500 4-channel digital waveform acquisition card. The Pixie-500 digitizer is designed specifically for fast radiation detectors and has high precision capabilities. The Pixie-500 was operated using the IGOR Pro control program. The PVT samples were coupled to a PMT (both the 2.54-cm PMT used with the analog electronics, and a Hamamatsu R329-02 5.08-cm diameter PMT were used for the measurements performed with the digital system) with optical grease and the PMT was connected to the Pixie-500. No preamplifier or amplifier was used with the digital electronics. The raw digitized waveforms were stored and used for post processing.

\subsection{Neutron Sources}

Three neutron sources were used for these measurements; two of the neutron sources were ${ }^{252} \mathrm{Cf}$ and the third source was AmBe. All of the neutron sources were purchased from Isotope Products Laboratory (IPL). One of the ${ }^{252} \mathrm{Cf}$ sources (source "A") was calibrated by IPL on December $15^{\text {th }}, 2003$, the other ${ }^{252}$ Cf source (source "B") was calibrated by IPL on September $1^{\text {st }}, 2011$, and the AmBe source was calibrated by IPL on February $11^{\text {th }}, 2003$. The assigned 
PNNL identification numbers and activities at the time of calibration and for the test dates are provided in Table 1.

Table 1: Neutron sources used for this measurement campaign.

\begin{tabular}{|c|c|c|c|}
\hline Source & ${ }^{252} \mathrm{Cf}$ "A" & ${ }^{252} \mathrm{Cf}$ "B" & AmBe \\
\hline PNNL ID & $56595-130 \mathrm{E}$ & I3-549 & $56595-180 \mathrm{~A}$ \\
\hline $\begin{array}{l}\text { IPL Reported } \\
\text { Source Activity }\end{array}$ & $20.0 \pm 3 \mu \mathrm{Ci}$ & $19.3 \pm 3 \mu \mathrm{Ci}$ & $23.0 \pm 3 \mathrm{mCi}$ \\
\hline $\begin{array}{c}\text { Activity } \\
10 / 21 / 2011\end{array}$ & $2.6 \pm 0.4 \mu \mathrm{Ci}$ & $\mathrm{N} / \mathrm{A}^{*}$ & $22.7 \pm 2.97 \mathrm{mCi}$ \\
\hline $\begin{array}{l}\text { Neutron Flux } \\
\text { 10/21/2011 }\end{array}$ & $\begin{array}{c}1.1 \times 10^{4} \pm 1.7 \times 10^{3} \\
\mathrm{n} / \mathrm{s}\end{array}$ & $\mathrm{N} / \mathrm{A}^{*}$ & $\begin{array}{c}5.6 \times 10^{4} \pm 8.4 \times 10^{3} \\
n / s\end{array}$ \\
\hline $\begin{array}{c}\text { Activity } \\
12 / 08 / 2011\end{array}$ & $2.5 \pm 0.4 \mu \mathrm{Ci}$ & $\mathrm{N} / \mathrm{A}^{*}$ & $22.7 \pm 2.97 \mathrm{mCi}$ \\
\hline $\begin{array}{c}\text { Neutron Flux } \\
\text { 12/08/2011 }\end{array}$ & $\begin{array}{c}1.1 \times 10^{4} \pm 1.7 \times 10^{3} \\
n / s\end{array}$ & $\mathrm{~N} / \mathrm{A}^{*}$ & $\begin{array}{c}5.6 \times 10^{4} \pm 8.4 \times 10^{3} \\
n / s\end{array}$ \\
\hline $\begin{array}{c}\text { Activity } \\
01 / 26 / 2012\end{array}$ & $2.4 \pm 0.4 \mu \mathrm{Ci}$ & $\mathrm{N} / \mathrm{A}^{*}$ & $22.7 \pm 2.97 \mathrm{mCi}$ \\
\hline $\begin{array}{l}\text { Neutron Flux } \\
\text { 01/26/2012 }\end{array}$ & $\begin{array}{c}1.0 \times 10^{4} \pm 1.6 \times 10^{3} \\
\mathrm{n} / \mathrm{s}\end{array}$ & $\mathrm{N} / \mathrm{A}^{*}$ & $\begin{array}{c}5.6 \times 10^{4} \pm 8.4 \times 10^{3} \\
n / s\end{array}$ \\
\hline $\begin{array}{c}\text { Activity } \\
02 / 20 / 2012\end{array}$ & $\mathrm{~N} / \mathrm{A}^{*}$ & $17.0 \pm 2.6 \mu \mathrm{Ci}$ & $\mathrm{N} / \mathrm{A}^{*}$ \\
\hline $\begin{array}{c}\text { Neutron Flux } \\
\text { 02/20/2012 }\end{array}$ & $\mathrm{N} / \mathrm{A}^{*}$ & $\begin{array}{c}7.2 \times 10^{4} \pm 1.1 \times 10^{4} \\
\mathrm{n} / \mathrm{s}\end{array}$ & $\mathrm{N} / \mathrm{A}^{*}$ \\
\hline
\end{tabular}

* No measurement performed on that date

The ${ }^{252} \mathrm{Cf}$ source " $\mathrm{A}$ " was used in its bare configuration and in a polyethylene and lead pig. The ${ }^{252} \mathrm{Cf}$ source "B" was used in its polyethylene and lead pig only. The AmBe source was used in its bare configuration and in a lead pig.

\subsection{Gamma Ray Sources}

Three gamma ray sources were used during this testing, ${ }^{137} \mathrm{Cs},{ }^{22} \mathrm{Na}$ and ${ }^{60} \mathrm{Co}$. All three sources were purchased from IPL; the source activities reported by IPL were measured on June $1^{\text {st }} 2003$. The PNNL assigned identification numbers and source activities at the time of calibration and for the test dates are listed in Table 2.

\footnotetext{
${ }^{1}$ The conversion used to obtain the neutron emission rate from the ${ }^{252} \mathrm{Cf}$ source was: $10 \mathrm{ng}$ of ${ }^{252} \mathrm{Cf}=5.4 \mu \mathrm{Ci}=2.3 \times 10^{4} \mathrm{n} / \mathrm{s}$ (Martin 1999). The conversion used to obtain the neutron emission rate from the AmBe source was: $6.6 \times 10^{-5} \mathrm{n} /(\mathrm{s} * \mathrm{~Bq})(\mathrm{Gibson}$ 1985).
} 
Table 2: Gamma ray sources used for the testing.

\begin{tabular}{|c|c|c|c|}
\hline Source & ${ }^{\mathbf{1 3 7}} \mathbf{C s}$ & ${ }^{{ }^{\mathbf{2 2}} \mathbf{N a}}$ & ${ }^{{ }^{\mathbf{0}} \mathbf{C o}}$ \\
\hline PNNL ID & $780-22-17$ & $971-80-6$ & $971-96-8$ \\
\hline $\begin{array}{c}\text { Source Activity } \\
\text { Reported by IPL }(\boldsymbol{\mu C i})\end{array}$ & $9.3 \pm 1.4$ & $9.6 \pm 1.4$ & $9.3 \pm 1.4$ \\
\hline $\begin{array}{c}\text { Activity 10/21/2011 } \\
\text { and 12/08/2011 }(\boldsymbol{\mu C} \mathbf{)})\end{array}$ & $7.7 \pm 1.2$ & $1.0 \pm 0.2$ & $3.10 \pm 0.5$ \\
\hline $\begin{array}{c}\text { Gamma Ray Flux } \\
\mathbf{1 0} \\
\mathbf{1 2} / \mathbf{2 1} / \mathbf{2 0 1 1} \text { and }\end{array}$ & $2.4 \times 10^{5} \pm 3.6 \times 10^{4}$ & $3.7 \times 10^{4} \pm 5.6 \times 10^{3}$ & $2.2 \times 10^{5} \pm 3.3 \times 10^{4}$ \\
\hline
\end{tabular}

${ }^{2}$ The branching ratios for the conversions used were ${ }^{137} \mathrm{Cs}: 0.85,{ }^{22} \mathrm{Na}: 0.99,{ }^{60} \mathrm{Co}: 1.99$ (Chu et al. 1999). 


\section{Equipment and Setup}

\subsection{Analog Electronics}

The analog measurements were made with the PMT (and PVT sample) located in a light-tight aluminum box. The PVT sample and the PMT were also wrapped in electrical tape to further reduce any noise from optical photons. The high voltage for the PMT was set at $800 \mathrm{~V}$ (based on previous experience with the PMT) and allowed to stabilize (in the light-tight environment) for approximately 30 minutes. The amplifier coarse gain was set to 10 , the fine gain was set to 0.3 , and the unipolar amplifier output (positive input polarity) was connected to the linear input of the Ortec 458 pulse shape analyzer (PSA). The time range of the PSA was $0.8 \times 1 \mu$ s and the input discriminator was $1,10 \mathrm{~V}$. The upper and lower limits of the PSA were set at 7 and 6, respectively, selected based on the spectra produced. With the upper level set to 7 and the lower level set to 6 two distinct signals (one from the gamma rays and one from the neutrons) were produced. The PSA output was connected to the PMCA which binned the data that collected and stored on a computer.

\subsection{Digital System}

The same aluminum box used for the analog measurements was used to house the PMT and PVT sample (coupled with optical grease and wrapped in electrical tape) for the measurements with the digital system. The output from the PMT was connected directly to the XIA Pixie-500. Two different base settings for the Pixie-500 were used for the measurements. Some of the settings were varied slightly but the same initial settings were loaded for each test. The two settings, referred to as $\mathrm{A}$ and $\mathrm{B}$, are as follows:

Table 3: PIXIE-500 settings used for data acquisition with the PVT sample.

\begin{tabular}{|l|l|l|}
\hline Parameter & A & B \\
\hline Trigger Rise Time $(\boldsymbol{\mu s})$ & 0.104 & 0.048 \\
\hline Trigger Flat Top $(\boldsymbol{\mu s})$ & 0.104 & 0.048 \\
\hline Trigger Threshold $(\boldsymbol{\mu s})$ & 6.0 & 25.0 \\
\hline Energy Filter Rise Time $(\boldsymbol{\mu s})$ & 0.208 & 0.224 \\
\hline Energy Filter Flat Top $(\boldsymbol{\mu} \mathbf{s})$ & 1.808 & 0.176 \\
\hline Waveform Trace Length $(\boldsymbol{\mu s})$ & 4.0 & 4.0 \\
\hline Waveform Trace Delay $(\boldsymbol{\mu s})$ & 1.0 & 1.0 \\
\hline Gain (set through oscilloscope) & 3.0 & 3.0 \\
\hline
\end{tabular}

Sample 1 was tested only with the A setting, Sample 2 was tested with both the A and B settings. For both sets of settings the energy reconstruction in the Pixie-500 was set to Integrator $=1$ (the 
gap-sum mode in Igor Pro), which sums all of the channels within the waveform length (note that there are other energy reconstruction options available, but the pulses are too fast for the other options to be effective). Although the gap-sum mode must be used to record the scintillator pulses it should be noted that the data analysis was performed using the raw pulse traces, not the integrated pulses.

\subsection{Measurements}

Measurements were made with shielded and bare neutron sources, shielded and unshielded gamma ray sources, and the neutron and gamma ray sources simultaneously. The shielding used was polyethylene, lead, tungsten, and steel. The majority of the neutron measurements were made with a 5.08-cm thick lead brick to reduce the intensity of the gamma rays (especially with the ${ }^{252} \mathrm{Cf}$ source).

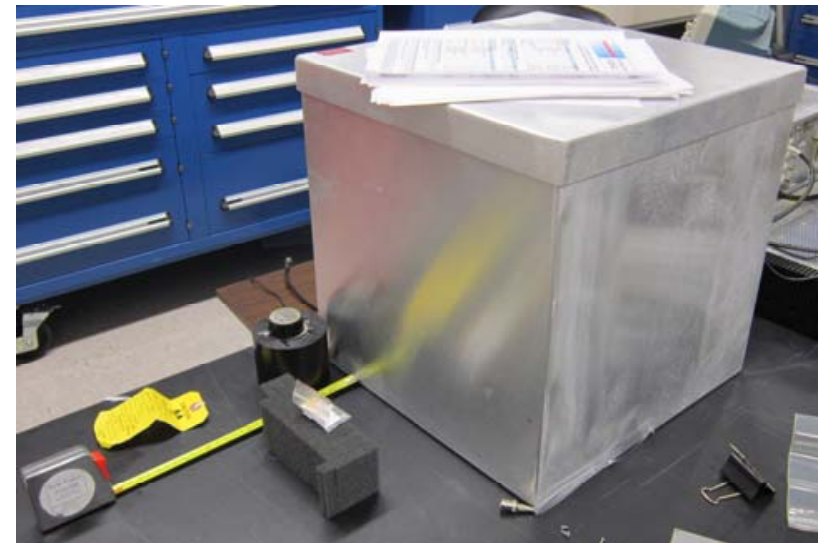

(a)

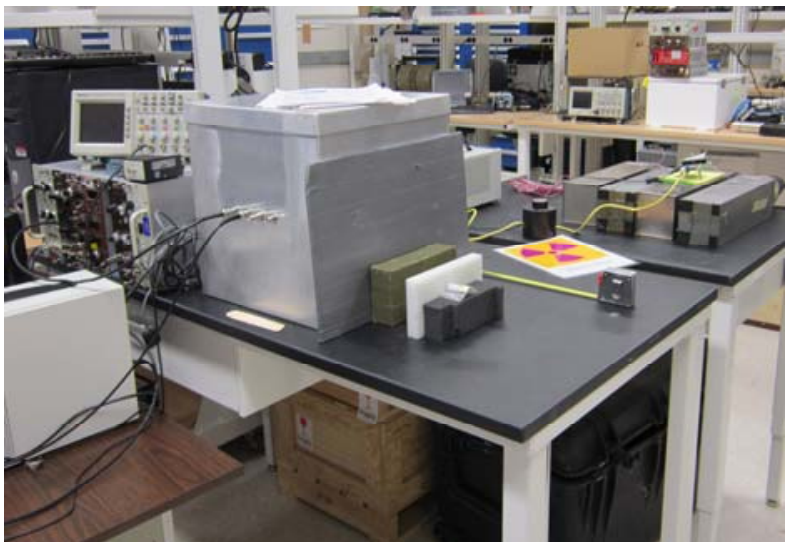

(b)

Figure 2: Digital experimental setup with a bare source and no shielding (a) and with a lead brick and poly shielding (b).

The PVT sample was positioned approximately $1 \mathrm{~cm}$ from the inside of the aluminum box (the outside of the tape wrapping the sample was just touching the inside of the box, but the tape was not wrapped tightly against the sample surface) and the center of the sample was approximately $8.25 \mathrm{~cm}$ above the base of the box. For all of the measurements with Sample 1 the sources were positioned $7 \mathrm{~cm}$ from the front of the tape wrapped sample (to account for the lead brick) at a height of approximately $8 \mathrm{~cm}$ (corresponding to the center of the sample). For the measurements with Sample 2 the source was positioned $15 \mathrm{~cm}$ from the front face of the box (these measurements were made in conjunction with another test campaign that required additional shielding configurations). 


\section{Results and Data Analysis}

\subsection{Analog}

The response of Sample 1 to both a neutron and a gamma ray source was measured with an analog circuit prior to data acquisition with the digital system; the initial analog system measurements were conducted to verify that gamma ray-neutron discrimination could be achieved with these samples. The analog data was collected with an un-moderated ${ }^{252} \mathrm{Cf}$ source shielded with $\mathrm{Pb}$ and $\mathrm{W}$ (to reduce the gamma ray background), and a bare ${ }^{137} \mathrm{Cs}$ source. The response of the sample to the bare ${ }^{252} \mathrm{Cf}$ source was compared to the response of the sample to the ${ }^{137} \mathrm{Cs}$ source to verify that one of the two peaks recorded with the ${ }^{252} \mathrm{Cf}$ source was due to gamma rays, and the other to neutrons. It was clearly demonstrated, as shown in Figure 3, that the neutron source produced a second peak that was not evident with the gamma ray source only.

The recorded neutron detection rate with the source located at two different distances from the detector $(10.1$ and $19.9 \mathrm{~cm})$ was used to make intrinsic neutron detection efficiency measurements. The source positions were selected to be large enough that the point source approximation could be applied. An intrinsic neutron detection efficiency of approximately $1 \%$ $(0.9 \%$ at $10.1 \mathrm{~cm}$ and $1.1 \%$ at $19.9 \mathrm{~cm})$ was calculated based on the counts recorded in the neutron peak at each distance. 


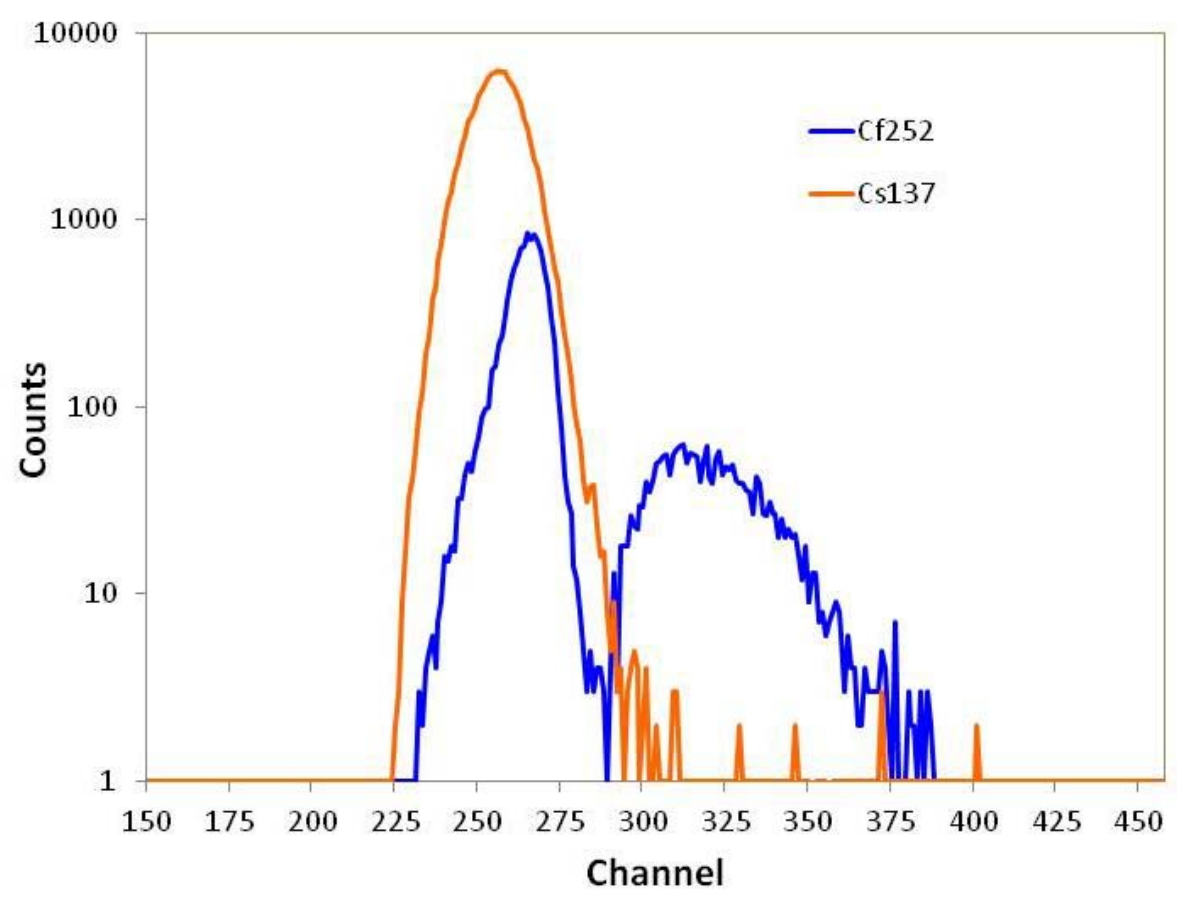

(a)

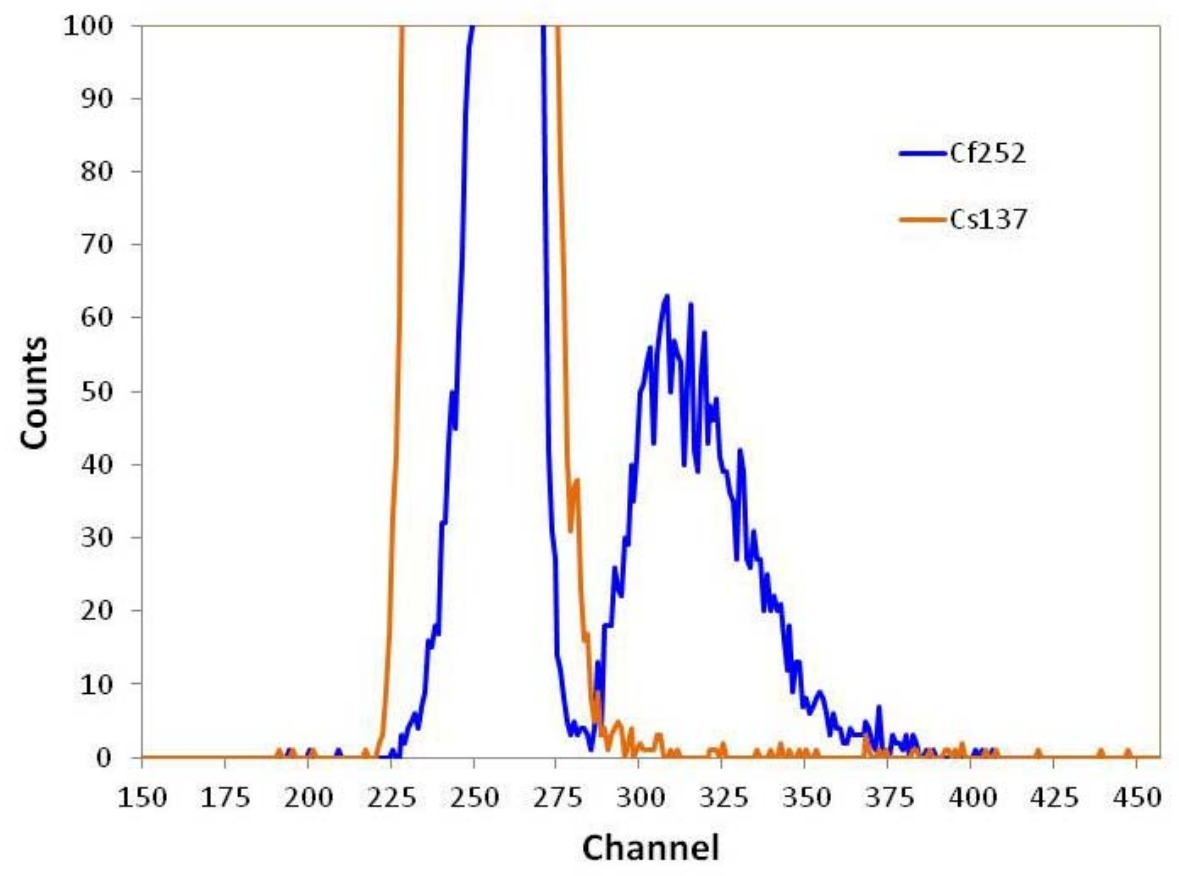

(b)

Figure 3: Analog pulse shape data from the PVT sample with a ${ }^{252} \mathrm{Cf}$ source and a ${ }^{137} \mathrm{Cs}$ source. The results are shown on a log scale (a) to show the complete set of data (note that the channels with zero counts could not be shown on the log plot) and zoomed in on a linear scale (b) which was used for the data analysis. 


\subsection{Digital}

Digital measurements were made with both PVT samples using the Pixie-500 data acquisition system. The data acquisition was performed using List Mode runs, which collect and store data on an event by event basis. The digital sampling rate of the Pixie-500 system is $500 \mathrm{MHz}$, so the time interval between the samples was 2 ns. The List Mode data for each run was stored and then post-processed with a MatLab script that analyzed the individual pulses. The neutron data shown in Section 5 was acquired with ${ }^{252} \mathrm{Cf}$ sources (source A un-moderated, and source B moderated) and $5.08 \mathrm{~cm}$ of $\mathrm{Pb}$ shielding. The $\mathrm{Pb}$ shielding was used to decrease the number of gamma rays incident on the detector and provide an equivalent test configuration to what was used by the developers (LLNL) when they provided their initial project overview results (Zaitseva 2011b). Measurements were also made with no Pb shielding, but the high number of gamma rays incident upon the detector produced extremely large data files in short amounts of time; the large data files made the data analysis more challenging. An example of the bare ${ }^{252} \mathrm{Cf}$ results collected with Sample 1 is shown in Appendix A. The samples' responses to the AmBe neutron source are also provided in Appendix A.

\subsubsection{Sample 1}

All of the ${ }^{252} \mathrm{Cf}$ data acquired with Sample 1 was obtained using source A with no moderation (i.e., no polyethylene, just the lead shielding). After the data was collected the post-processing MatLab script was used to identify (read) and store the individual pulses. Examination of the individual pulses (Figure 4) illustrated that two shapes were present. The pulse in Figure 4a is in response to a gamma ray and the pulse in Figure $4 \mathrm{~b}$ is in response to a neutron; the same pulses are plotted on a log scale in Figures $4 \mathrm{c}$ and $4 \mathrm{~d}$ (gamma ray and neutron, respectively) to better illustrate the differences in the pulse tails. The longer tail in the neutron pulse compared to the gamma ray pulse was utilized to establish the separation of the two signals. The ratio of the total charge produced by the incident radiation (the area under the pulse trace) to the charge in the "tail" portion of the pulse (the Qratio) was plotted as a function of the peak height. The channel ranges for the pulse peak and the pulse tail were set in the MatLab code; for most of the analysis the peak was set from channel 0 to 35 and the tail was set from channel 35 to 250 (these windows are discussed further in Section 5.2.3). A histogram of the data in the Qratio versus peak height plot, with the data summed along the y-axis, was created to provide a plot of the count rate (the total summed points were divided by the system live-time) versus the Qratio. The histogram was used to calculate the FOM and quantify the gamma ray-neutron separation. 


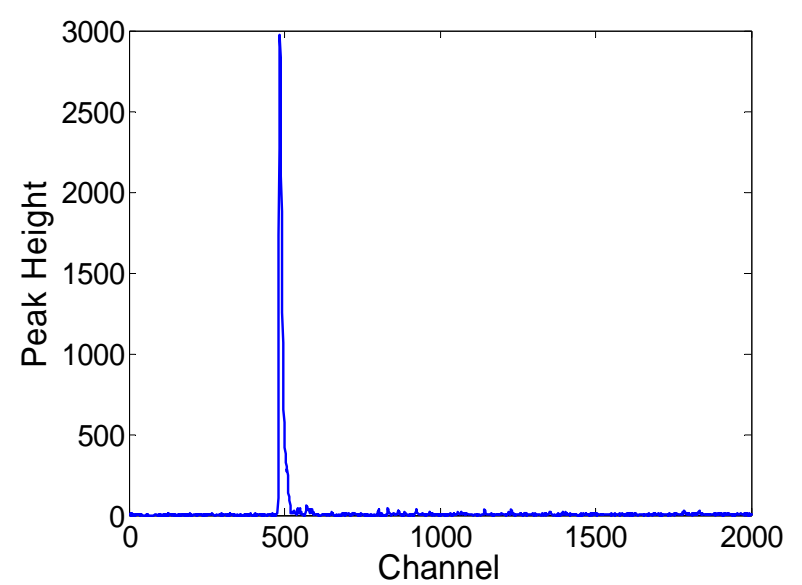

(a)

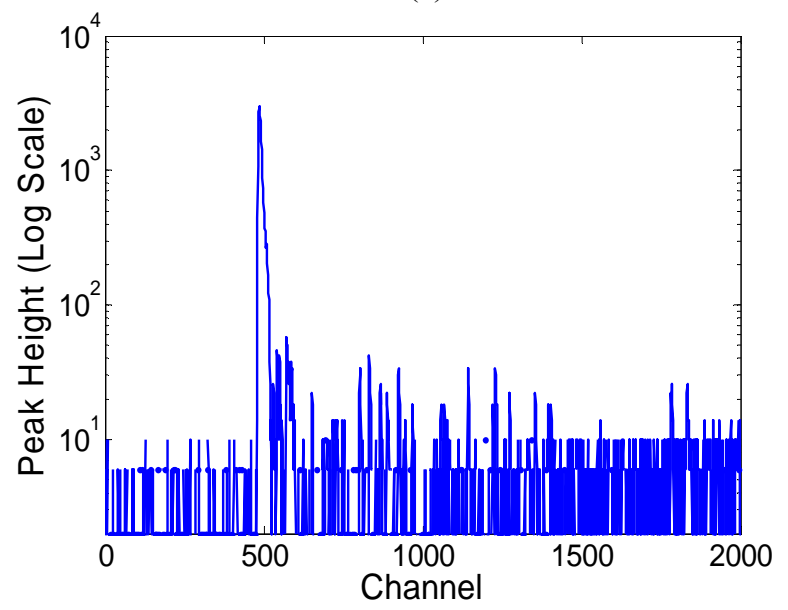

(c)

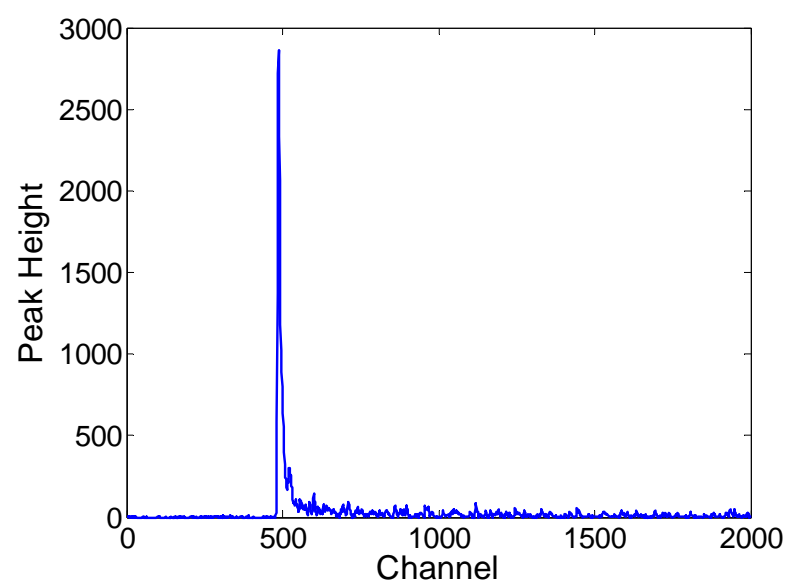

(b)

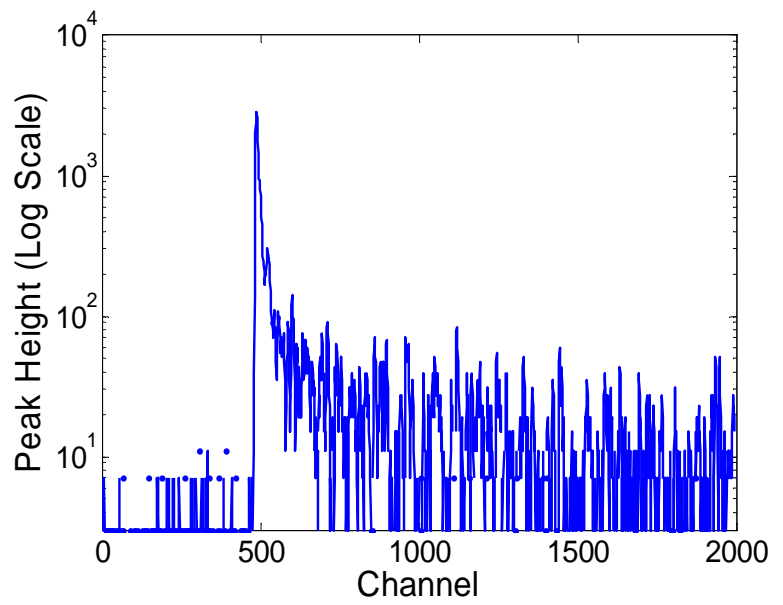

(d)

Figure 4: Individual gamma ray (a) and neutron (b) pulses recorded with Sample 1 in response to a ${ }^{252} \mathrm{Cf}$ source. The same pulses are shown on a log scale (gamma ray (c) and neutron (d)) to illustrate the different tail shape.

The first Qratio versus peak height plot showed very poor gamma ray-neutron separation (Figure 5). Similarly, the 1-D histogram of the data (Figure 6) showed no discernible peaks, and a FOM could not be calculated. The significant difference in the shape of the individual pulses generated by gamma rays and neutrons, and the two distinct signals recorded with the analog electronics, indicated that a more pronounced separation of the gamma ray and neutron signals should have been produced. To determine if there were discrepancies in the recorded pulses that would cause the blurring noted in the Q-ratio versus peak height plot several individual pulse traces were overlaid on top of each other. The initial plot (Figure 7) appeared acceptable, but a closer examination of the pulses (zoomed in) demonstrated that there was a slight time jitter that produced inconsistent Q-ratio calculations for windows of the same channel width (Figure 8). 


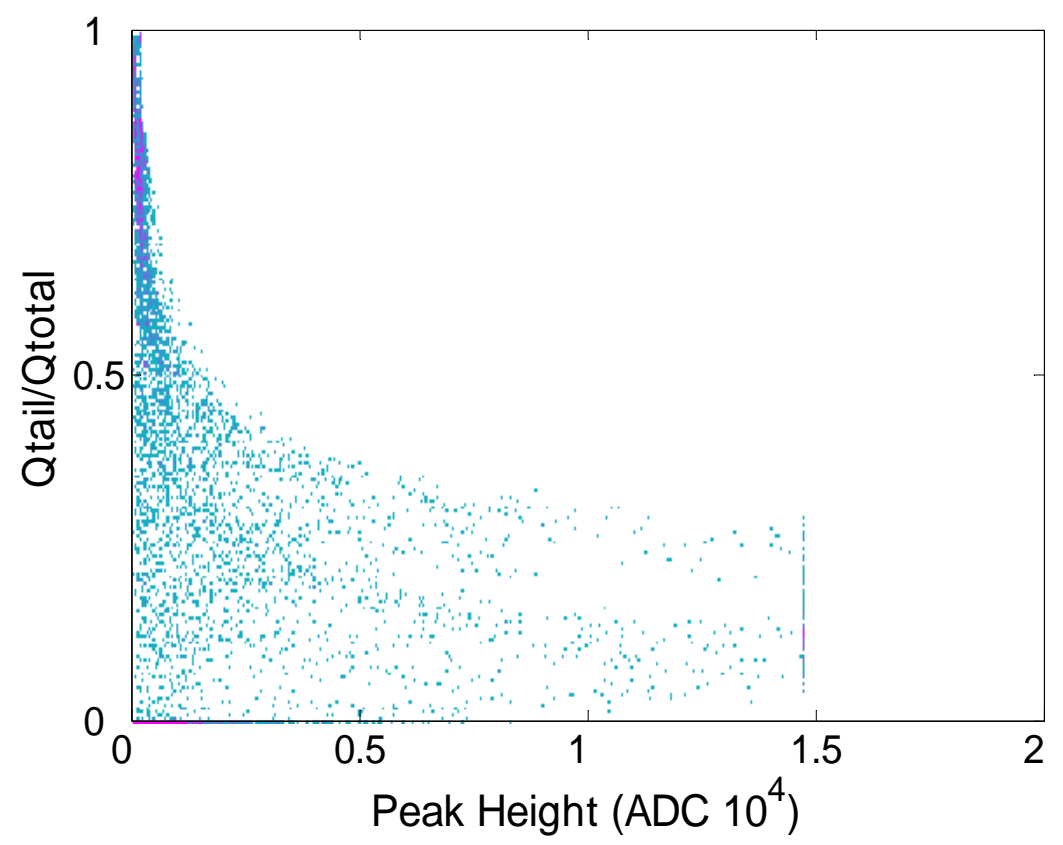

Figure 5: The ratio of the charge in the tail of the pulses to the total charge associated with the pulse versus peak height obtained from Sample 1 with a bare ${ }^{252} \mathrm{Cf}$ source shielded by $5.08 \mathrm{~cm}$ of $\mathrm{Pb}$. Note that a "neutron region" can be identified for peak heights greater than 0.5 and with Qratios above approximately 0.25 but there is not a clear gamma ray-neutron distinction.

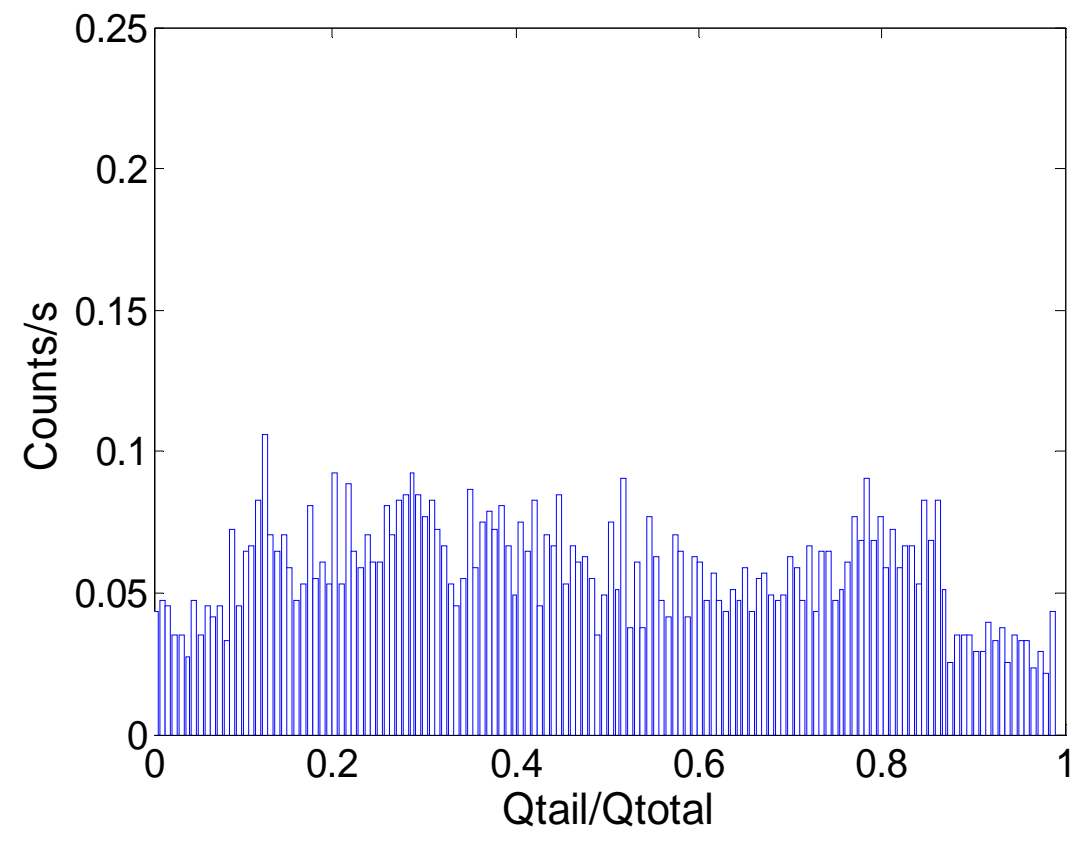

Figure 6: Histogram of the Qratio data shown in Figure 5. Note the lack of clear gamma ray and neutron peaks. 


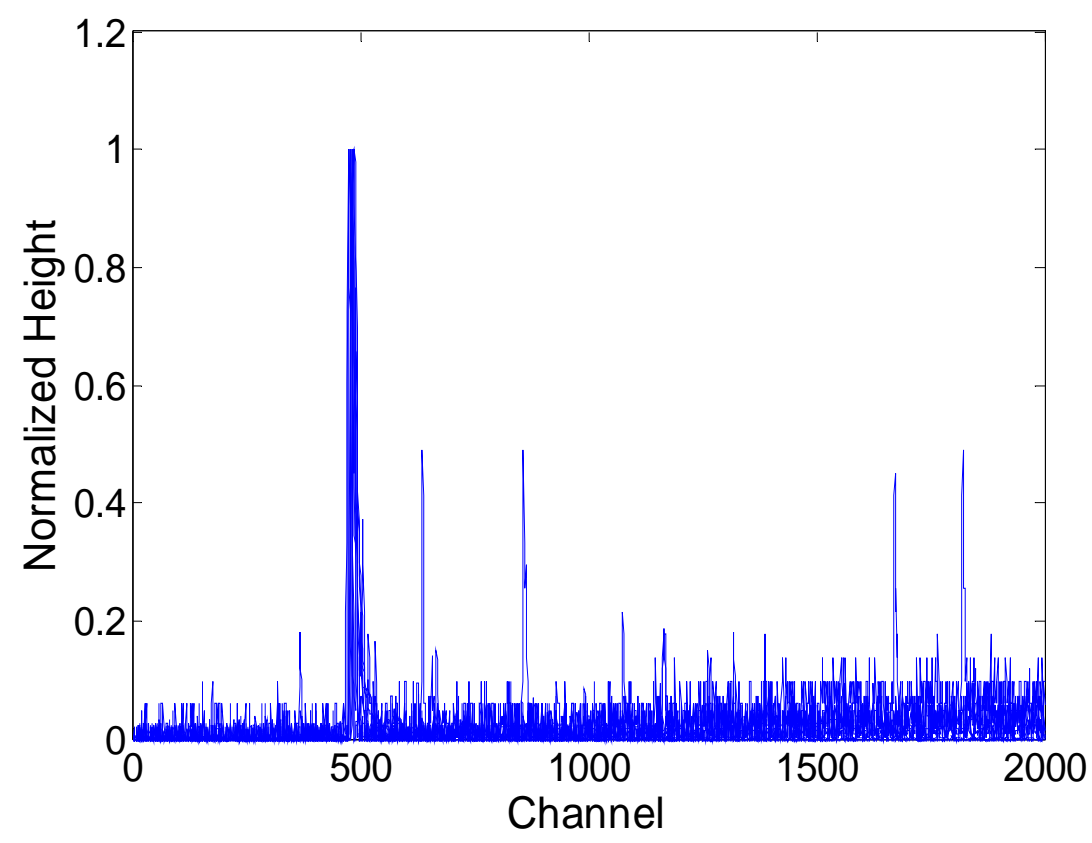

Figure 7: Ten over-laid traces recorded in response to a ${ }^{252} \mathrm{Cf}$ source with Sample 1.

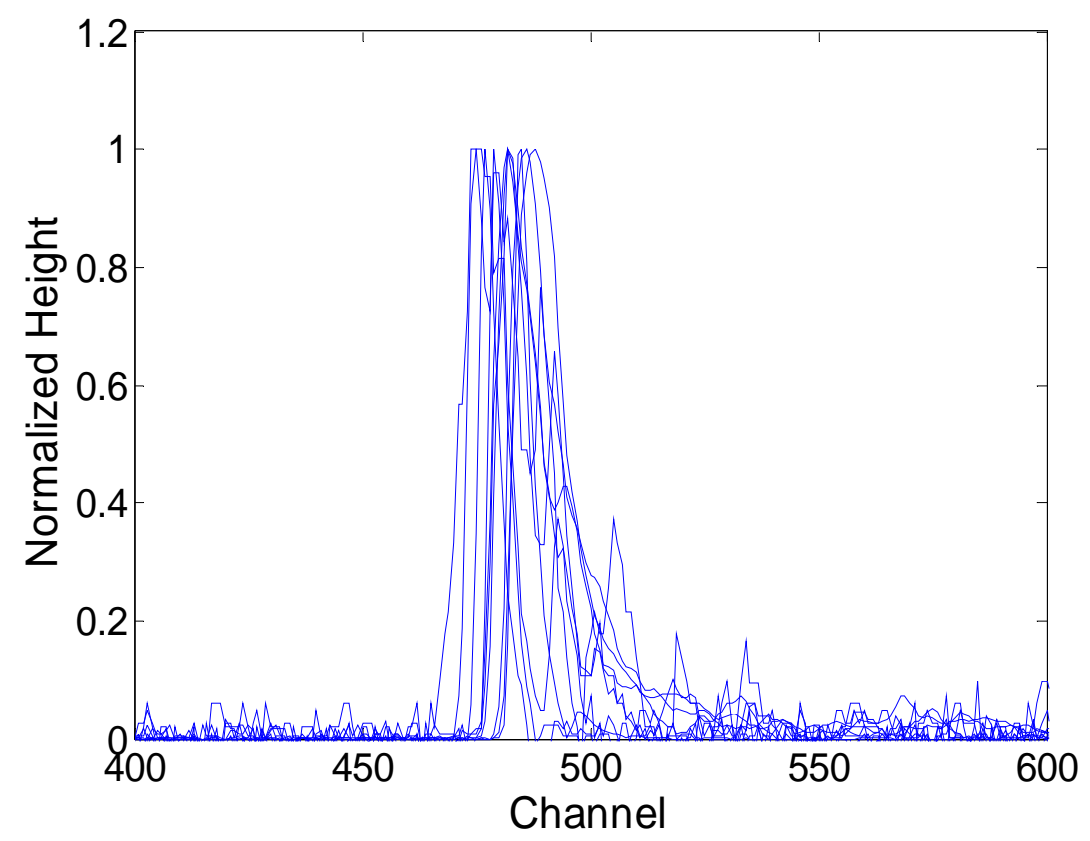

Figure 8: Detail of the pulses shown in Figure 7.

To overcome the slight time jitter inherent to the data acquisition system, which is evident in Figure 8 , the pulses were aligned prior to any analysis being performed. The aligned pulse comparison (Figure 9) appears similar to the unaligned pulses, until they are examined in more detail. A magnified image (of the x-axis) shows that the aligned peaks will have different Qratios (Figure 10). 


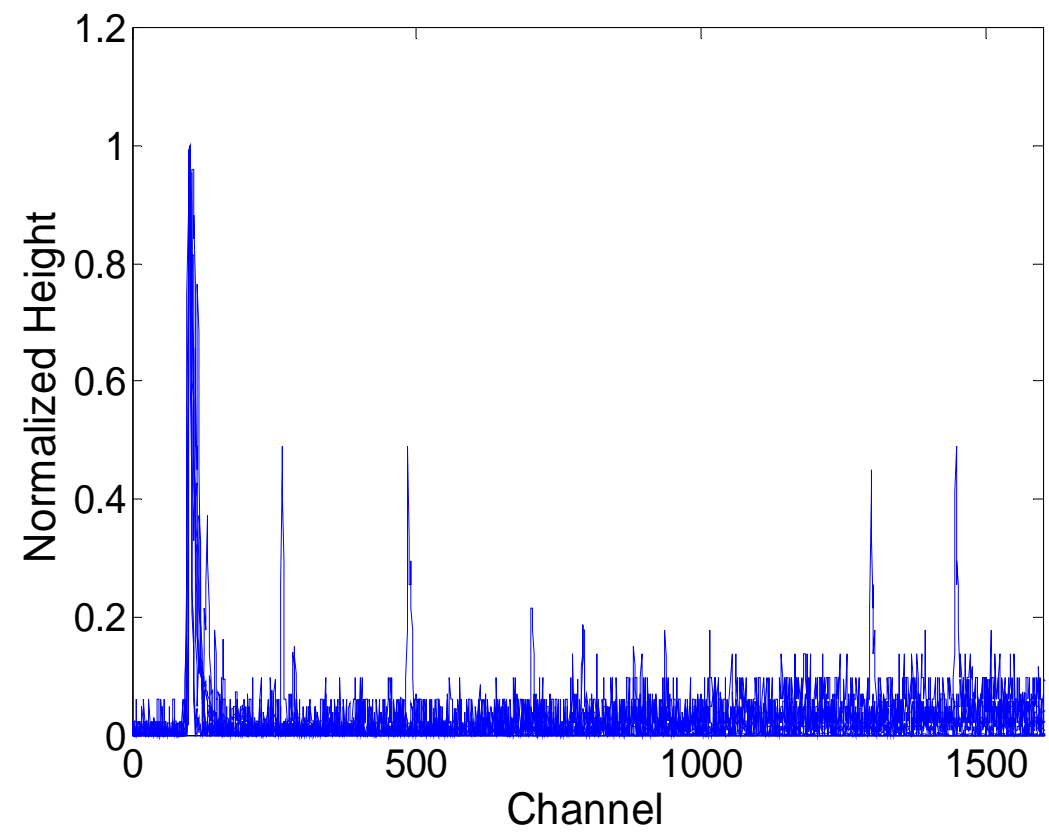

Figure 9: Ten over-laid pulses recorded in response to a ${ }^{252} \mathrm{Cf}$ source by Sample 1 with the maximum peak heights aligned.

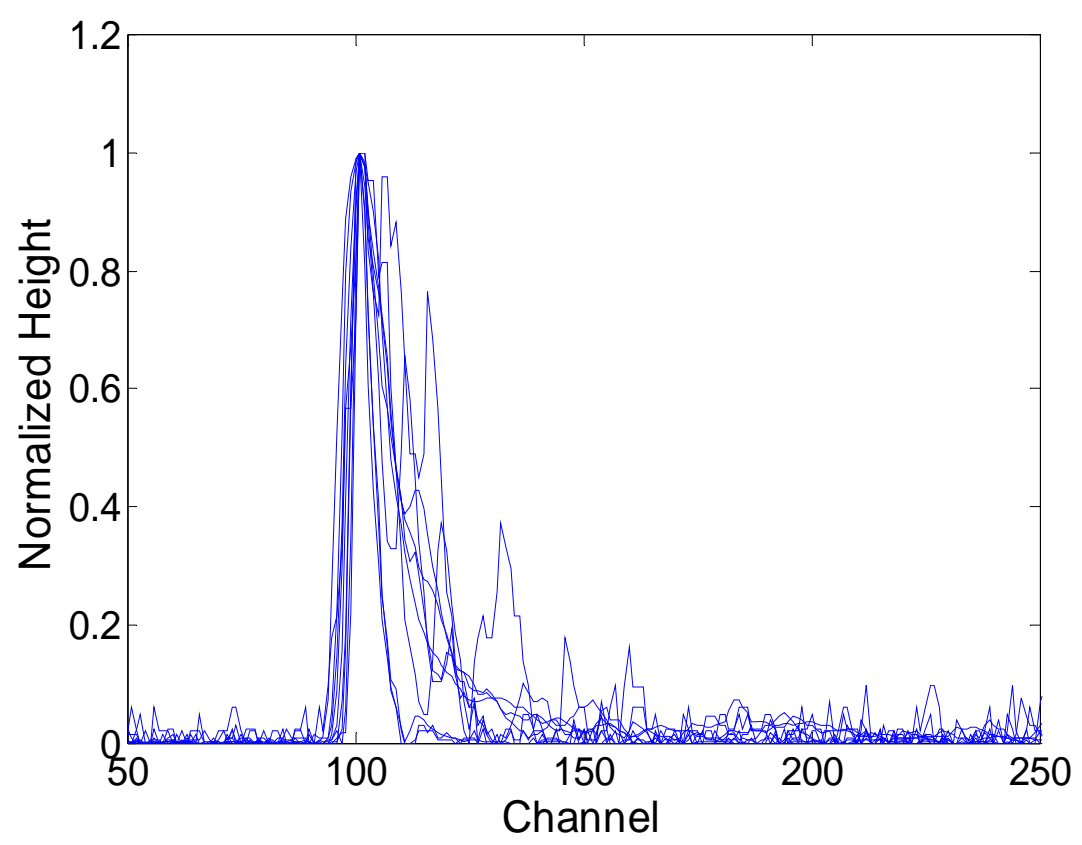

Figure 10: Detail of the pulses shown in Figure 9.

A plot of the Q-ratio versus the peak height with the aligned pulses (aligned and then background subtracted) demonstrates clear gamma ray-neutron separation (Figure 11). The separation was quantified by calculating the FOM from the histogram of the data (Figure 12). The FOM was calculated by using a Gaussian fit on each of the peaks (note, each Gaussian was fit separately using different parameters) to estimate the location of the center of the peak and the corresponding FWHM. The parameters selected for the Gaussian fit can change the FOM 
calculated, the effect of these parameters is discussed in Section 5.2.3. It should be noted that the Gaussian fit, as it is currently applied, works best on symmetrical data (i.e. when the histogram peaks are distributed uniformly). It is evident in Figure 12 that the Gaussian can only be fit over a limited region for certain distributions (those that are not true Gaussian distributions) using the method applied to these results. Smoothing the data, or an alternate algorithm for fitting the Gaussian, may produce improved fits. Estimates of the FWHM based on the peak height produced similar results to the quantitative fits (even when the entire peak couldn't be fit) and thus the quantitative method was used for the data presented in this report. The FOM calculated for the response of Sample 1 to the ${ }^{252} \mathrm{Cf}$ Source A, with the peaks aligned, was calculated to be 0.88 , which is only $69 \%$ of the FOM required for the detector to be considered a good gamma ray - neutron discriminator (as described in Section 2).

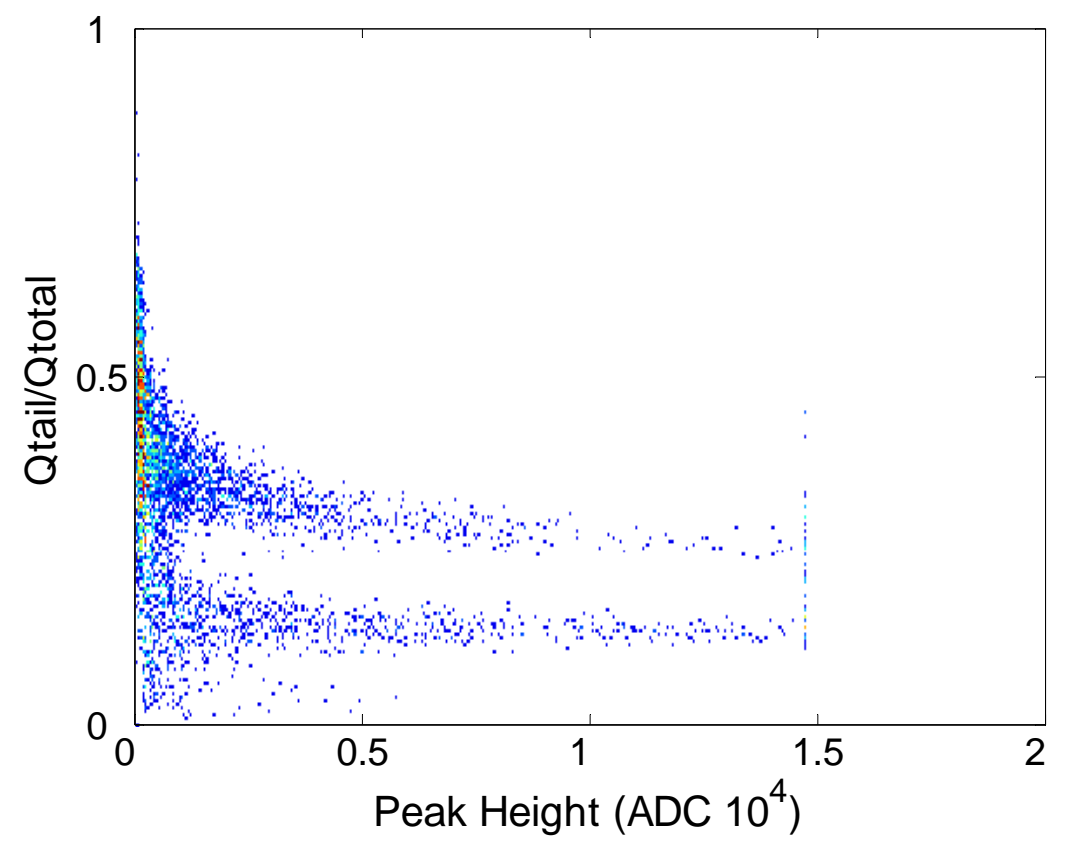

Figure 11: Ratio of the charge in the tail of the pulse to the total charge in the pulse as a function of the peak height, after the pulse peak height maximums were aligned. Note the clear neutron (higher) and gamma ray (lower) regions. 


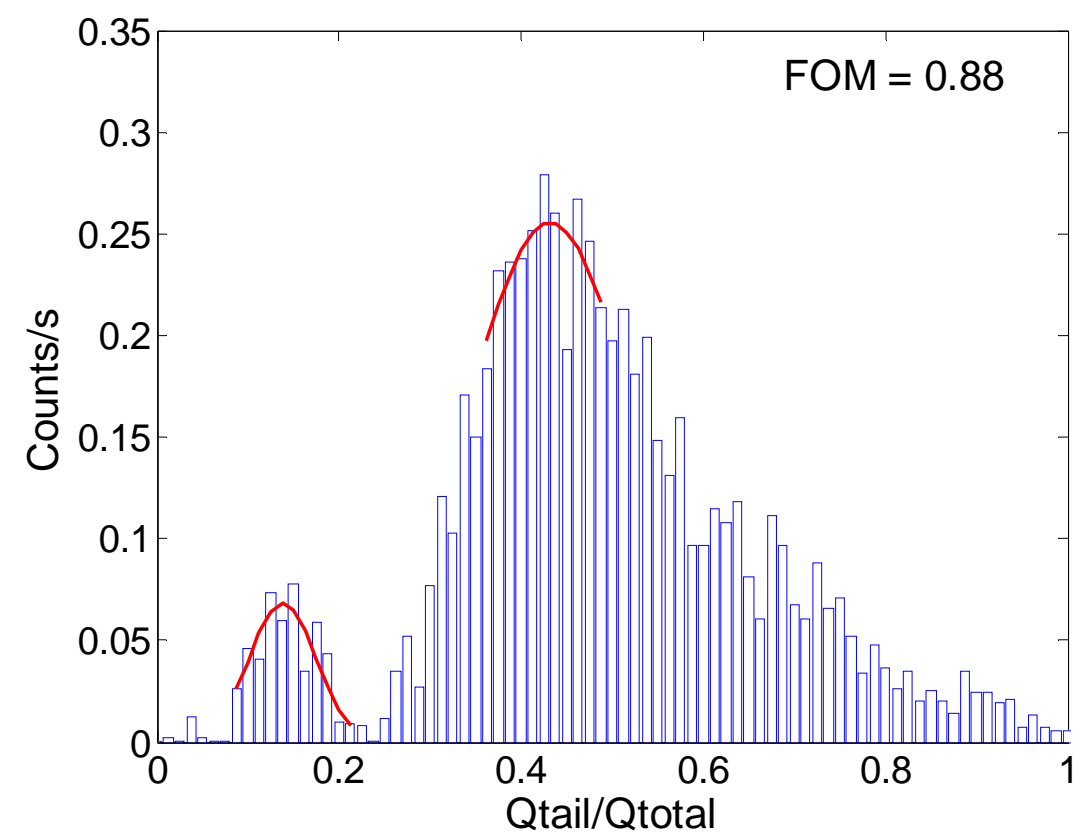

Figure 12: Histogram of the data in Figure 11. Note the clear gamma ray (lower Qratio) and neutron (higher Qratio) peaks.

The large amount of low peak height data (evident in Figure 11) was presumed to be indistinct signals produced by the data acquisition system triggering on low level pulses. The low peak height data was minimized by adding a lower level threshold during the post processing (the thresholds are discussed further in Section 5.2.3). An upper level threshold was also added to remove the pulses with peak heights above what could be processed. Initially measurements were made with a lower software gain set in the PIXIE-500 ( 1 instead of 3 ) to reduce the number of pulses with peak-heights above what could be processed. However, it was found that some of the pulses were still too large to be binned correctly, and with the lower gain setting more pulses were eliminated by the lower level threshold. Thus, it was determined that more pulses were available for analysis in the useful region with the higher software gain. The lower and upper peak height thresholds used for this analysis were 0.25 and 1.4 , respectively. The results achieved with the thresholds are shown in Figure 13 and Figure 14. The same thresholds were applied to the background data and the data collected when a source was present. The thresholds were applied prior to background subtraction (but after the pulses were aligned). The peak height thresholds selected can have a significant impact on the reported sample performance; this is further discussed in Section 5.2.3.

It should be noted that the peak in Figure 14 with the lower Qratio has a poor Gaussian fit; however, this did not affect the FWHM calculation as the same number of channels would included in the width at the half maximum height if a lower peak were identified and fit qualitatively. Also, the peak height is not a direct factor for the FOM calculation, so the high peak itself did not change the reported FOM. 


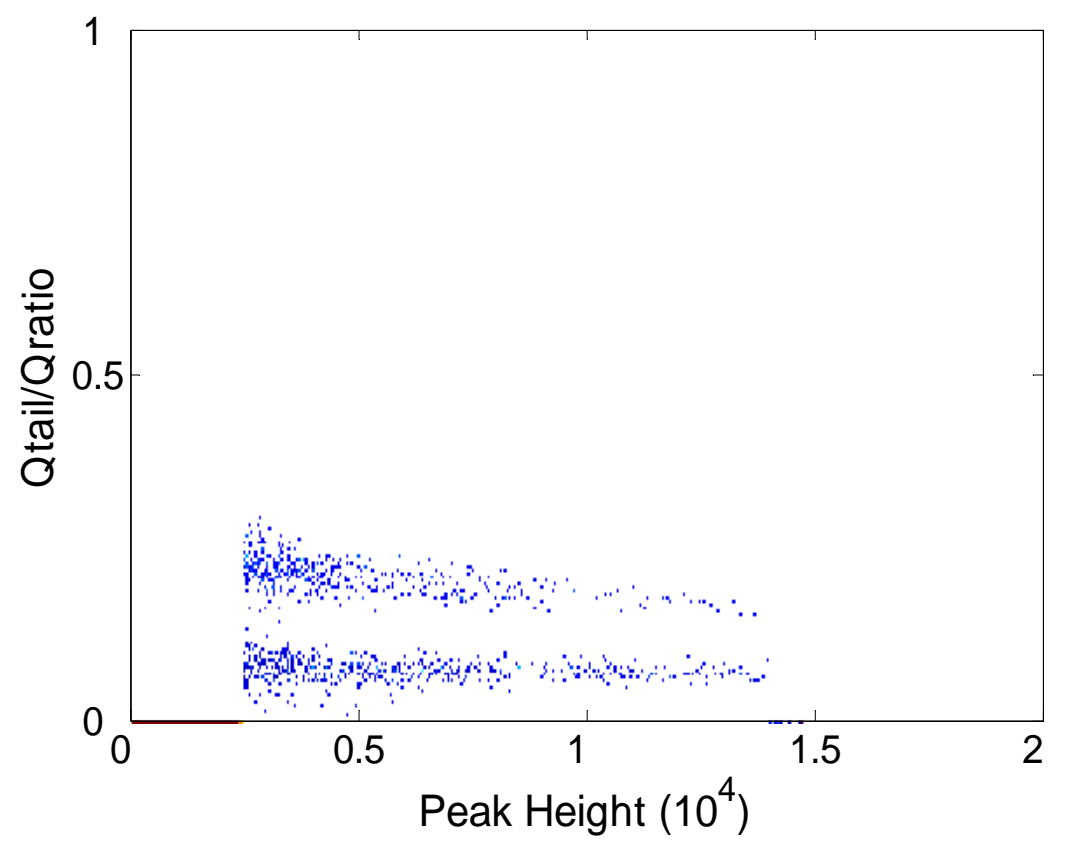

Figure 13: The same data plotted in Figure 11 with an upper and lower threshold (1.4 and 0.25 respectively) applied to the Qratio.

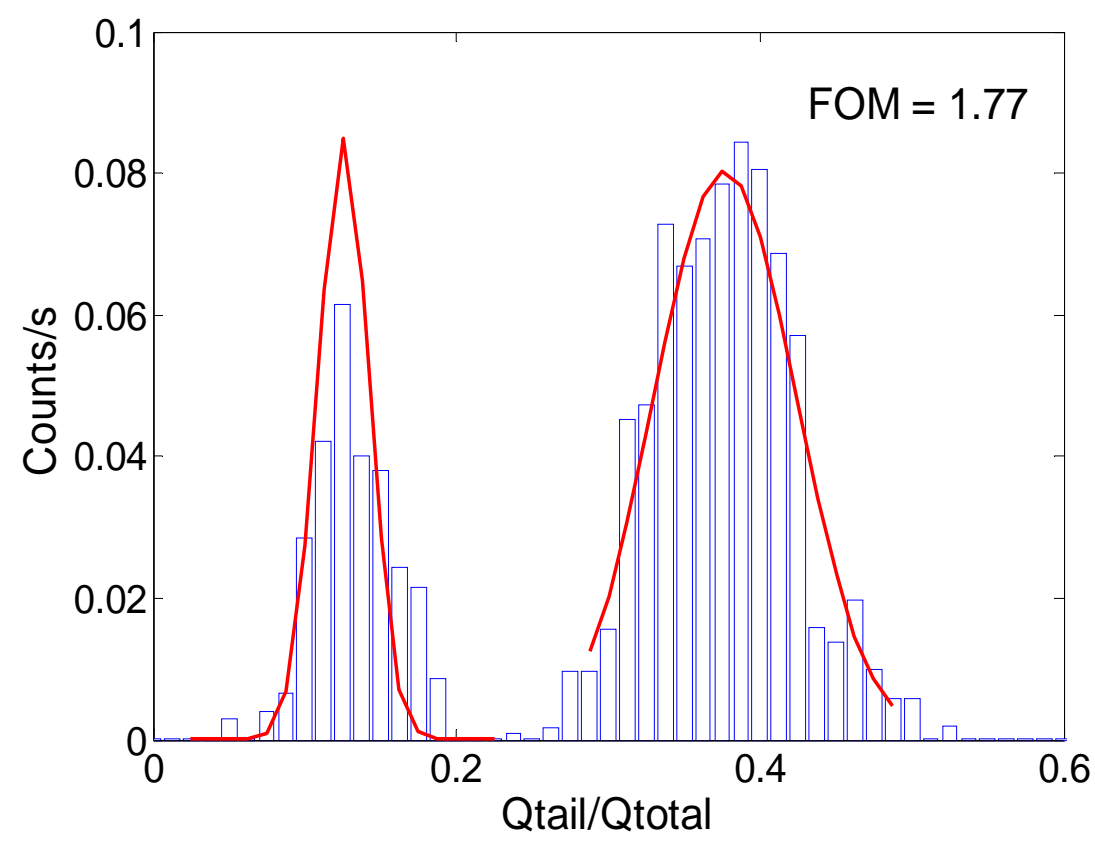

Figure 14: Histogram of the data in Figure 13.

The FOM calculated for the response of Sample 1 to the ${ }^{252} \mathrm{Cf}$ Source A after cutting the low and high data from the histogram was 1.77. This FOM is greater than the minimum FOM required for a detector to be considered capable of adequate gamma ray-neutron discrimination.

Data was also acquired with a gamma ray source only to verify that the two signals produced were indeed from neutrons and gamma rays (separately). Applying the same post processing 
techniques as described above (peak alignment and upper and lower thresholds) a Q-ratio versus peak height plot and a corresponding histogram were generated. The data from the ${ }^{137} \mathrm{Cs}$ clearly demonstrates only a single peak, as shown in Figure 15 and Figure 16, verifying that the second peak in the histogram of the data recorded in response to the ${ }^{252} \mathrm{Cf}$ source was due to neutrons.

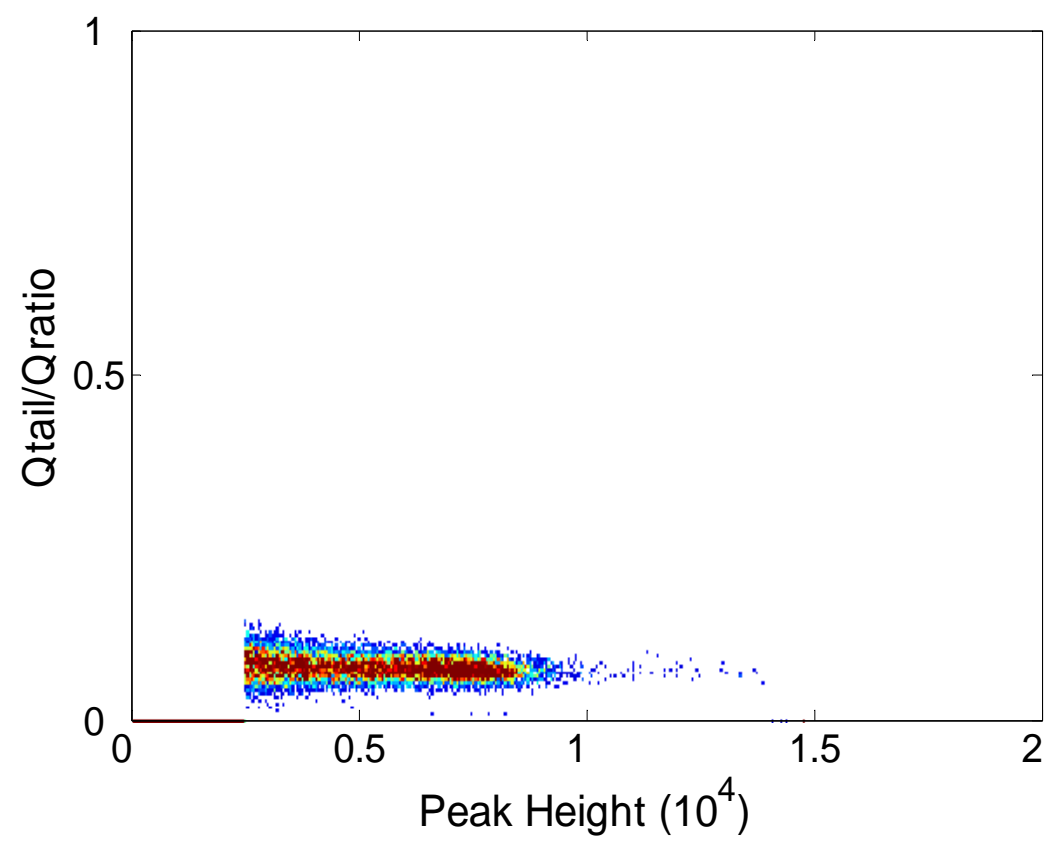

Figure 15: The Qratio as a function of peak height for the pulses recorded by Sample 1 in response to a ${ }^{137} \mathrm{Cs}$ source with the pulse peaks aligned and an upper and lower level threshold (1.4 and 0.25 respectively) applied.

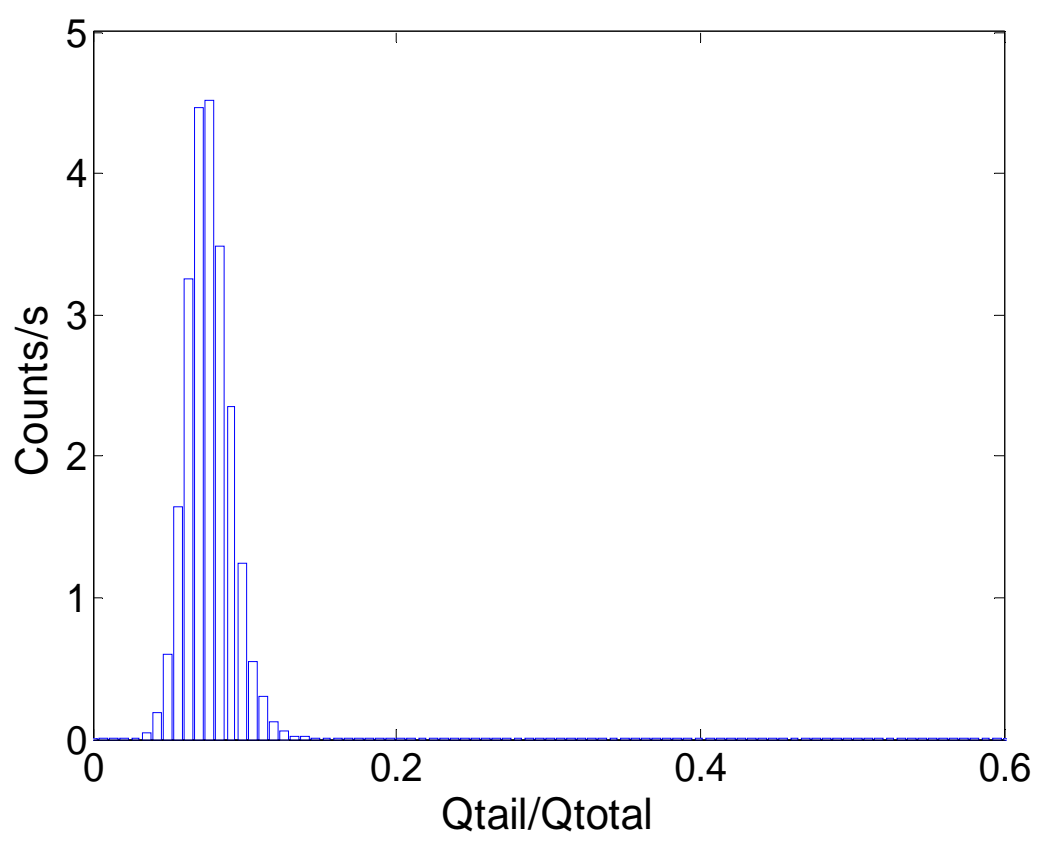

Figure 16: Histogram of the data in Figure 15. 


\subsubsection{Sample 2}

The same data acquisition setup was used to acquire data with the second sample. However, a stronger ${ }^{252} \mathrm{Cf}$ source, Source B, was used for these measurements so that higher count rates and better statistics could be achieved (in a reasonable amount of time). The source was located 15$\mathrm{cm}$ from the front of the box housing the sample (the increased distance was needed to accommodate some additional shielding configurations). The same data analysis techniques as used for Sample 1 (pulse alignment and upper and lower peak height thresholds) were applied to produce a plot of the Q-ratio versus peak height (Figure 17). A histogram of the data (Figure 18) was used to calculate the FOM. The Gaussian fit on the gamma ray peak underestimates the peak height. However, as in Figure 14, the number of channels assigned to the FWHM wouldn't change with a different peak height fit, and thus neither would the FOM. Additional data from Sample 2 is shown in Appendix A.

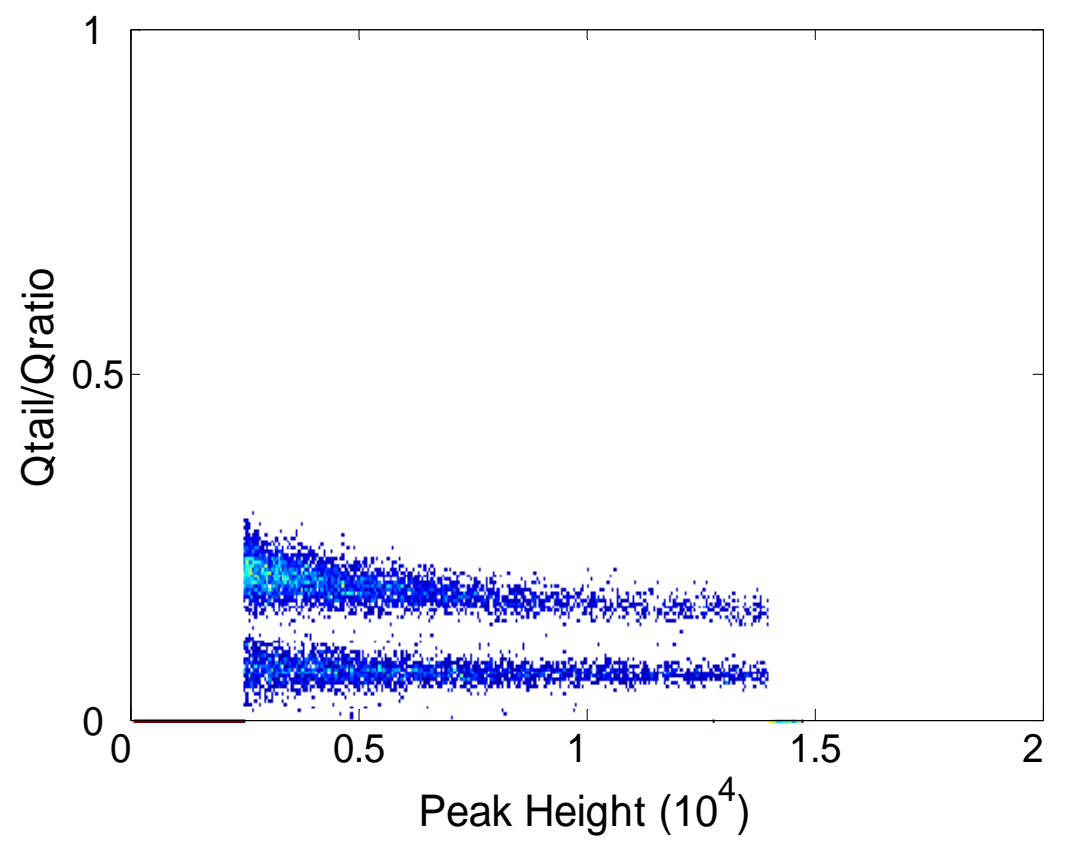

Figure 17: Qratio as a function of peak height for the aligned pulses recorded in response to ${ }^{252} \mathrm{Cf}$ by Sample 2 with an upper (1.4) and lower (0.25) threshold applied to the Qratio. 


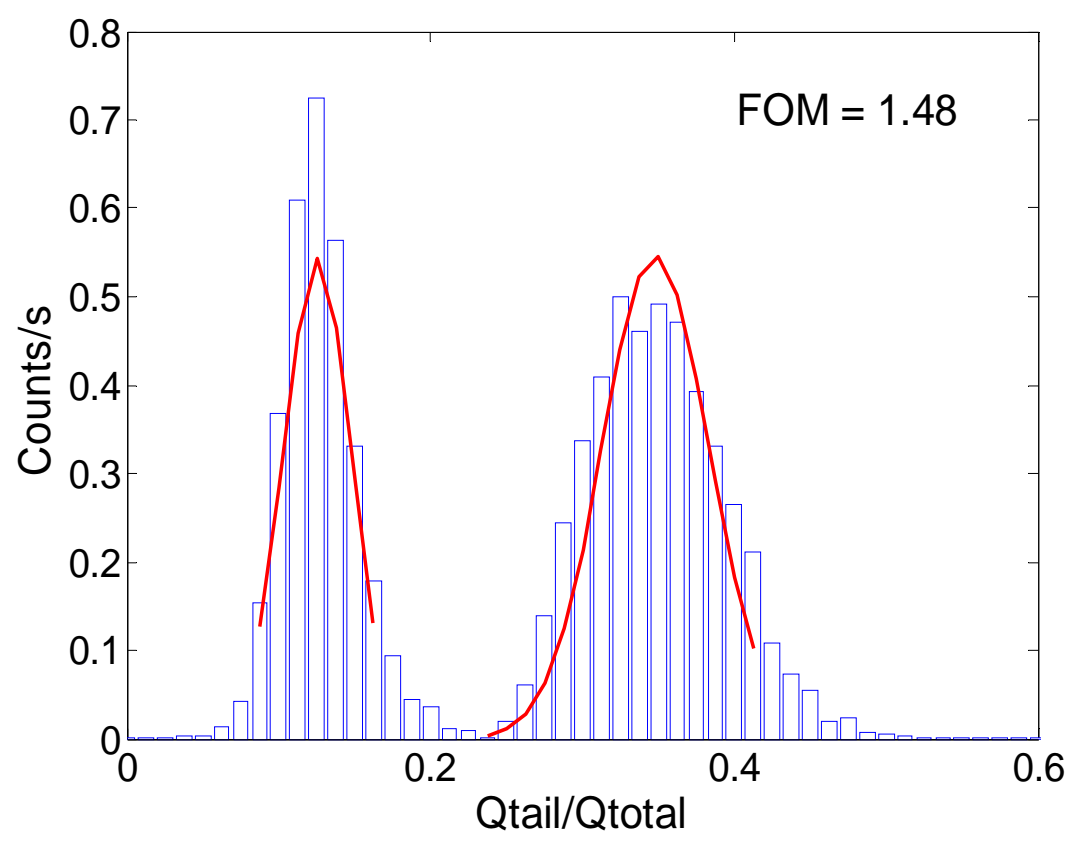

Figure 18: Histogram of the data from Figure 17.

The FOM calculated for the data collected with Sample 2 in response to the ${ }^{252} \mathrm{Cf}$ source was 1.48. The FOM achieved with Sample 2 was lower than the FOM calculated with Sample 1, but was still greater than the minimum FOM discussed in Section 2.

\subsubsection{Acquisition and Analysis Settings}

The selection of a threshold, either for accepting a pulse when collecting data or counting a pulse during analysis, can have a large impact on the results. There is a range of trigger thresholds that can be set for data acquisition with the PIXIE-500. If the threshold is set too low the system will trigger on noise, which will increase the dead-time and produce large data files which consist mostly of pulses that are useless for analysis. If the threshold is set too high the number of real pulses recorded is reduced, and the statistics are decreased. A comparison of the count rate as a function of the threshold (for Sample 2) is shown in Table 4. A threshold of 6 was used for data collection with Sample 1; however, for Sample 2 large dead-time was noted when the stronger ${ }^{252} \mathrm{Cf}$ source was used, so the threshold was set to 25 . 
Table 4: The event rates recorded with different threshold settings during 1 minute acquisitions with Sample 2 in response to the ${ }^{252} \mathrm{Cf}$ source shielded with 5.08-cm of $\mathrm{Pb}$.

\begin{tabular}{|c|c|}
\hline Threshold & Event Rate (cps) \\
\hline 2 & 12602 \\
\hline 4 & 67 \\
\hline 6 & 61 \\
\hline 8 & 51 \\
\hline 10 & 48 \\
\hline 12 & 43 \\
\hline 25 & 31 \\
\hline 35 & 27 \\
\hline
\end{tabular}

The peak height threshold selected during data analysis affects not only the count rate but also the FOM. As the lower level threshold is increased, the FOM is improved, as shown in Figure 19 for measurements made with Sample 2; however, the improved results are realized at the cost of the amount of data available for analysis. The standard method of calibrating the scintillator energy response to the Compton edge of the $662 \mathrm{keV}$ line from ${ }^{137} \mathrm{Cs}$ was not performed in this study, but the range of thresholds shown in Figure 19 gives the range of FOM values for comparison with other measurements.

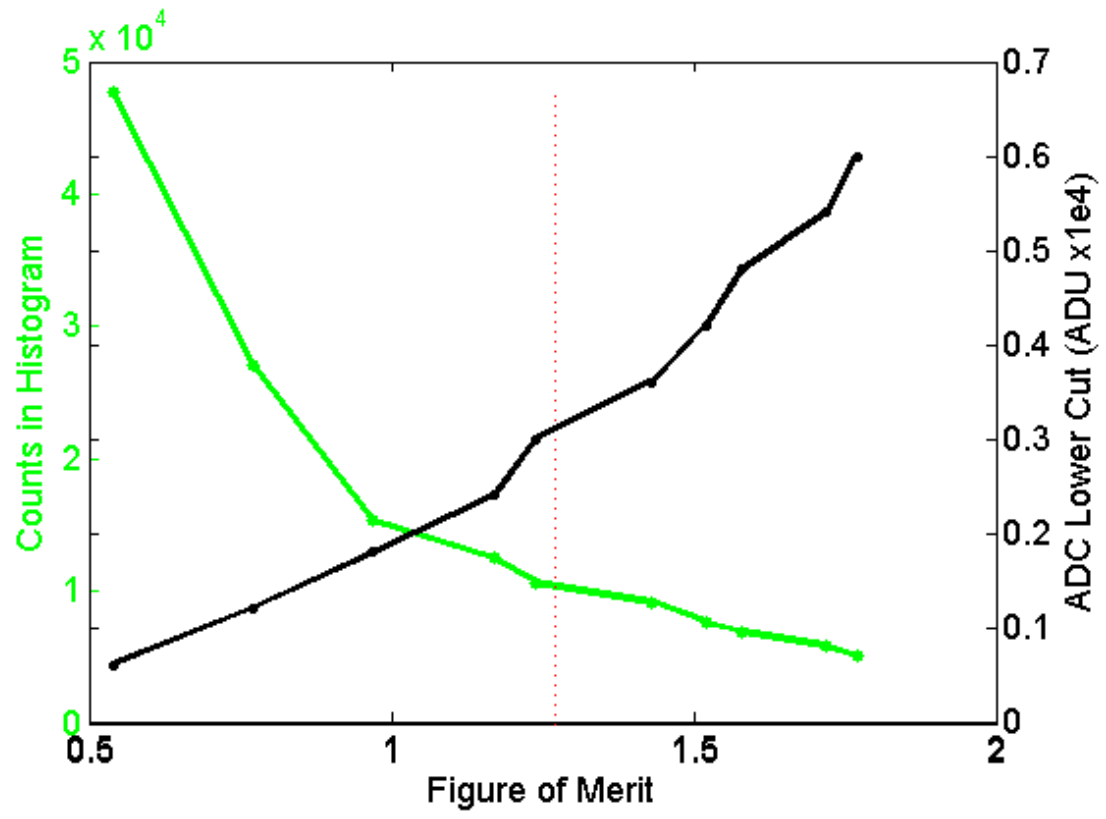

Figure 19: Lower level threshold, total number of counts, and FOM relationship. The red dotted line represents the commonly accepted minimum FOM required for adequate neutron-gamma ray separation.

In addition to the thresholds selected, the channel ranges used to define the total pulse and the pulse peak will also impact the FOM. The channels used for the peaks and for the total pulse affect which pulses are categorized as neutrons, and which pulses are categorized as gamma rays. 
Different channel ranges will also emphasize variations in different regions of the pulse. The optimal ranges for the windows in this data analysis were selected by calculating the FOM with several different sets of channel windows. The best performing peak and total channel ranges were selected by identifying the highest average FOM. Based on the results shown in Table 5 the highest average FOM was produced with a peak channel range of 0-35 and a total channel range of 0-250. Consequently, these are the windows that were used throughout this analysis.

Table 5: The affect of the windows used to define the pulse peak and the total pulse on the FOM calculated for each sample.

\begin{tabular}{|c|c|c|c|}
\hline Peak Channel Range & Total Channel Range & FOM Sample 1 & FOM Sample 2 \\
\hline $0-20$ & $0-100$ & 1.34 & 1.16 \\
\hline $0-20$ & $0-250$ & 1.70 & 1.51 \\
\hline $0-20$ & $0-300$ & 1.63 & 1.49 \\
\hline $0-35$ & $0-100$ & 1.02 & 0.85 \\
\hline $0-35$ & $0-250$ & 1.77 & 1.48 \\
\hline $0-35$ & $0-300$ & 1.70 & 1.41 \\
\hline $0-50$ & $0-100$ & 0.85 & 0.85 \\
\hline $0-50$ & $0-250$ & 1.44 & 1.42 \\
\hline $0-50$ & $0-300$ & 1.35 & 1.44 \\
\hline
\end{tabular}

There are other factors in the analysis that can affect the reported FOM for a sample. One of these factors is the fit of the Gaussian function used to determine the peak center and FWHM. The parameters of the Gaussian fit, such as the number of channels over which the function is fit, the center point for counting the channels, and the spread of the Gaussian, will all affect the calculated FOM. For example, simply reducing the number of channels in the Gaussian fit by one changed the FOM calculated for Sample 2 with windows of 0-35 and 0-250 from 1.48 to 1.34. The FOMs reported in Table 5 were all calculated with the Gaussian parameters optimized for those particular settings. However, there may be changes to the method used to fit the Gaussian that would improve the results. An additional consideration is the method used to align the pulses. For this analysis the pulse peaks were aligned to be in the same channel, however the pulses could be aligned to a specific threshold instead. Several thresholds (10\%, 25\% and 50\% of the peak height) were tested (the thresholds were applied to the normalized pulses before background subtraction), but it was found that a consistent improvement was not realized beyond aligning the peaks. The alignment is an additional parameter that could be modified on an individual basis and may result in an improvement in performance.

The thresholds, channel settings, selected Gaussian parameters, and pulse alignment must all be considered when comparing results. The Gaussian fitting routine, the upper and lower thresholds, and the non-Gaussian distribution of the Qratio will all contribute to the uncertainty of the FOM. 


\section{Conclusion}

The response of two fast neutron sensitive PVT samples was tested. Measurements were made with both analog and digital electronics. The intrinsic neutron detection efficiency was estimated, using the analog electronics, to be $\sim 1 \%$. Post processing of the digitized pulses was performed to generate histograms of the ratio of the charge in the tail of the pulse to the charge in the entire pulse. The histograms were used to calculate the FOM for the gamma ray-neutron separation. It was determined that to achieve an acceptable FOM the pulse peaks had to be aligned preceding analysis, and an upper and lower threshold had to be applied prior to generating a histogram of the data. The thresholds applied to this data were one-dimensional upper and lower cuts on the pulse heights. There was not a significant slope to the Qratios for the data presented here, so a two dimensional threshold was not applied. However, this is something that could be considered to reduce the noise in the histograms used to calculate the FOM.

The best (highest) FOM, was obtained with Sample 1, and was 1.77. There are additional measures that could be explored to improve the FOM, such as aligning the individual pulses to different thresholds (instead of the maximum height), changing the thresholds, and smoothing the data prior to fitting the Gaussian function.

The samples tested demonstrated fast neutron sensitivity and good (FOM > 1.27) gamma rayneutron separation. However, larger samples would be required for most practical applications. The larger samples would need to be tested to verify that the same level of performance obtained with the small samples could be achieved. 


\section{References}

Chu S., L. Ekstrom, and R. Firestone. 1999. Table of Radioactive Isotopes (Online Edition).

Gibson J., and E. Piesch. 1985. Neutron Monitoring for Radiological Protection. Technical Reports Series No. 252, International Atomic Energy Agency, Vienna.

Hamel N., V. Simic, and S. Normand. 2008. Fluorescent 1,8-naphthalimides-containing polymers as plastic scintillators. An attempt for neutron-gamma discrimination. Reactive and Functional Polymers, 68:12 1671-1681.

Knoll G. 2003. Radiation Detection and Measurement, $3^{\text {rd }}$ Ed., John Wiley and Sons.

Martin R., J. Knauer, and P. Balo. 1999. Production, Distribution, and Applications of Californium-252 Neutron Sources. Presented at: Industrial Radiation and Radioisotope Measurement Applications, October 1999.

Winyard R., J. Lutkin and G. McBeth. 1971. Pulse Shape Discrimination in Inorganic and Organic Scintillators. Nuclear Instruments and Methods, 95 141-153.

Zaitseva N., A. Glenn, L. Carman, R. Hatarik, S. Hamel, M. Faust, B. Schabes, N. Cherepy and S. Payne. 2011a. Pulse Shape Discrimination in Impure and Mixed Single-Crystal Organic Scintillators. IEEE Transactions on Nuclear Science. 58:6 3411-3420.

Zaitseva N., 2011b. Solution-Grown Organic Single Crystal Scintillators. SNM Movement Detection /Radiation Sensors and Advanced Materials Portfolio Review RadSensing 2011. LLNL-PRES-485517.

Zaitseva N., B.L. Rupert, I. Pawelczak, A. Glenn, H.P. Martinez, L. Carman, M. Faust, N. Cherepy, and S. Payne. 2012. Plastic Scintillators with Efficient Neutron/Gamma Pulse Shape Discrimination. Nuclear Instruments and Methods in Physics Research A, 668 88-93. 


\section{Appendix A}

\subsection{Additional Results Sample 1}

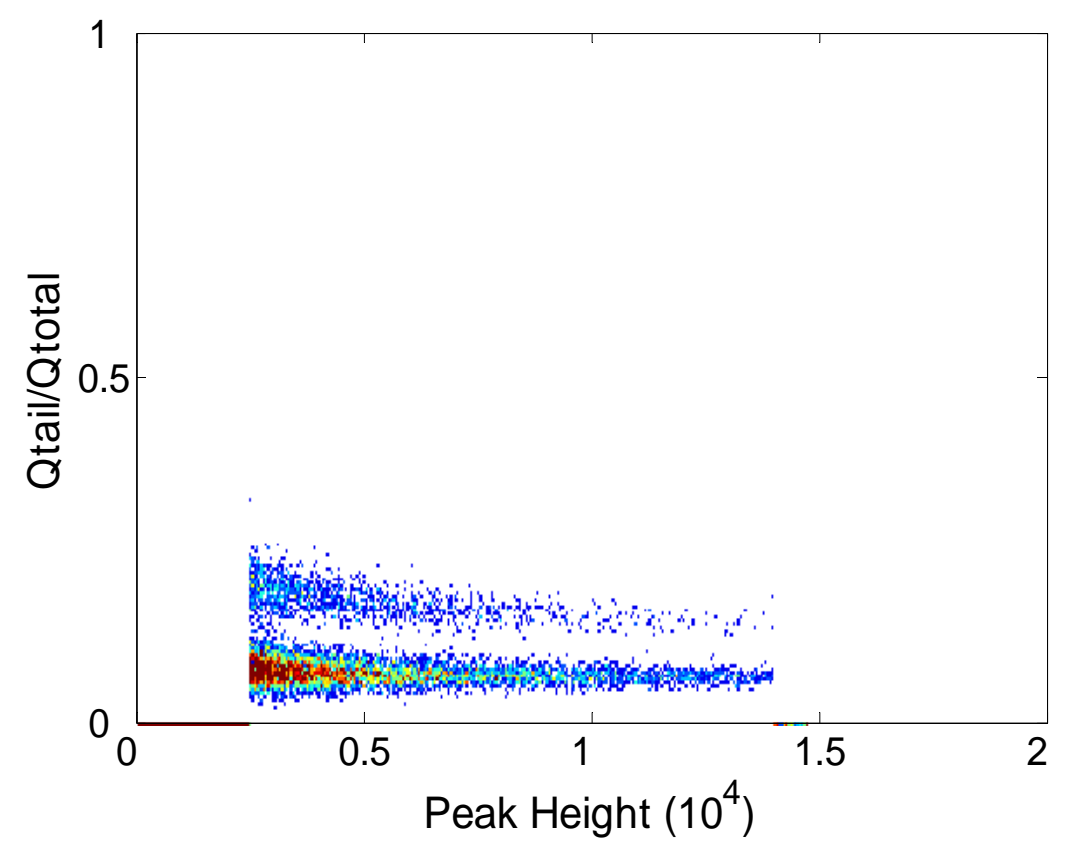

Figure 20: Results from the pulses recorded by Sample 1 in response to a bare ${ }^{252} \mathrm{Cf}$ source with the pulse peaks aligned and an upper (1.4) and lower (0.25) threshold applied.

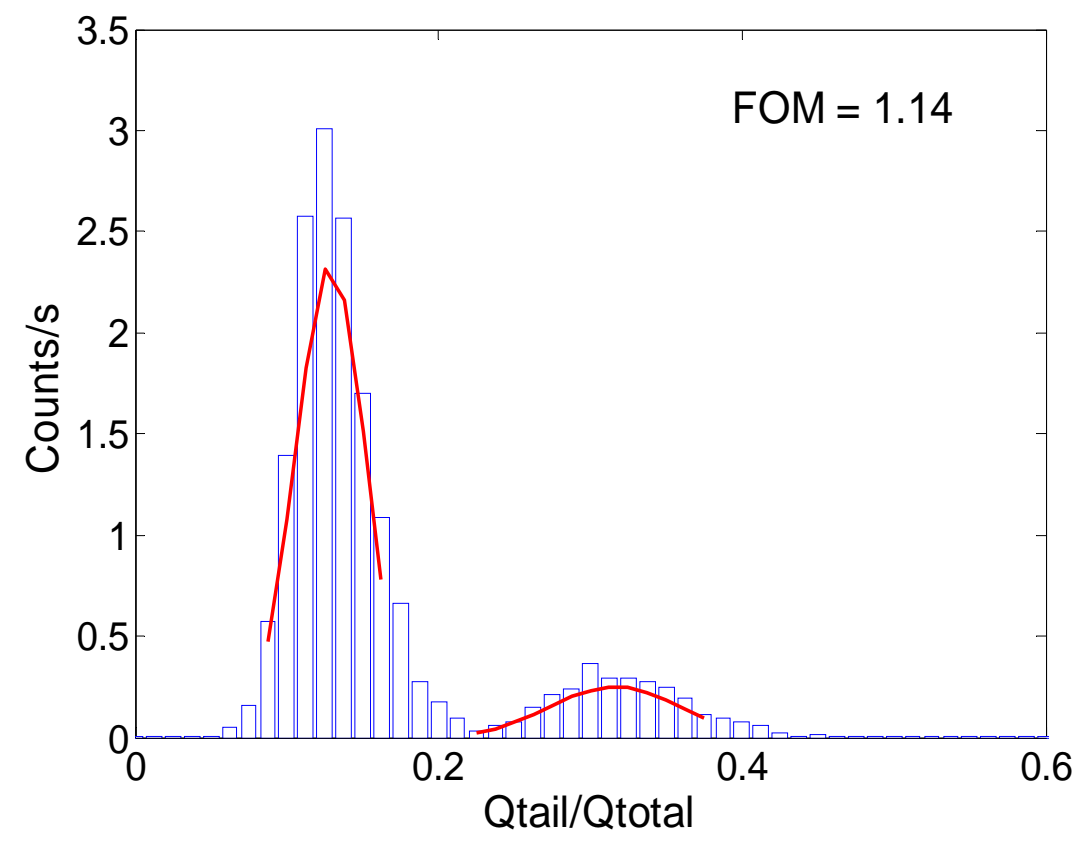

Figure 21: Histogram of the data in Figure 20. 
The FOM calculated for the bare ${ }^{252} \mathrm{Cf}$ source (located $7 \mathrm{~cm}$ from the front of the detector) with no lead shielding was 1.14 (the FOM with lead shielding was 1.74).

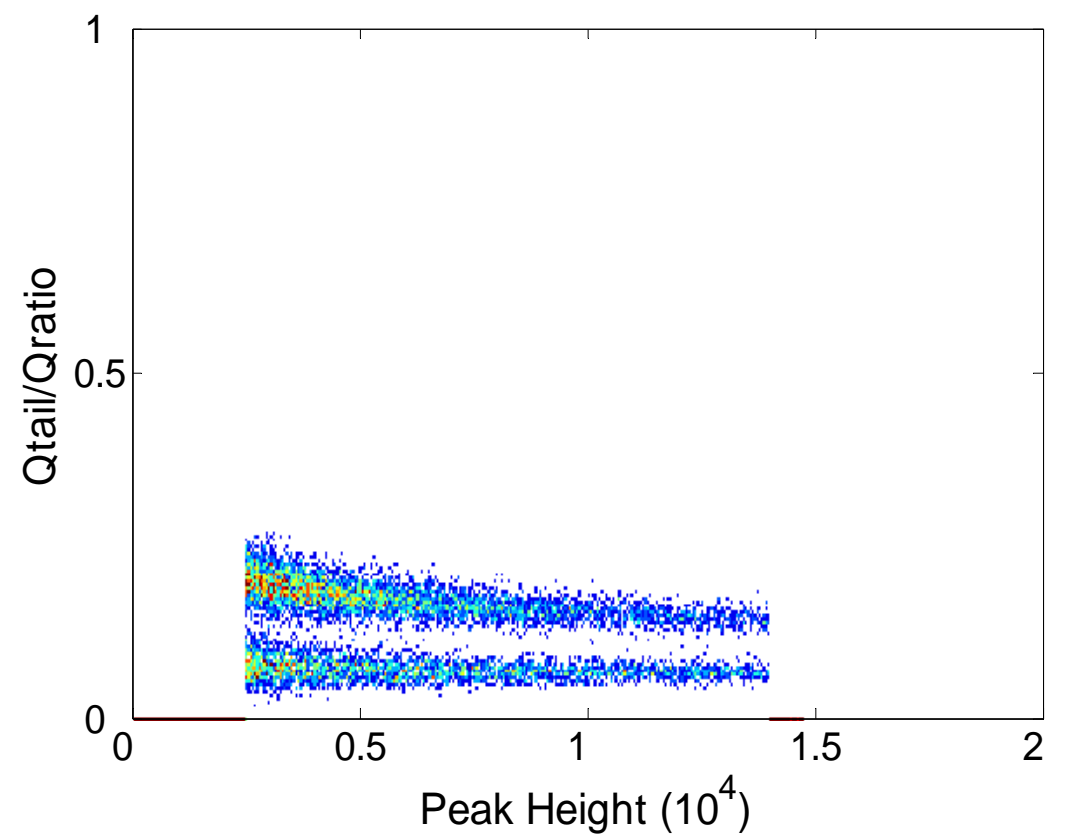

Figure 22: Results from the pulses recorded by Sample 1 in response to a bare AmBe source shielded by 5.08 $\mathrm{cm}$ of $\mathrm{Pb}$ with the pulse peaks aligned and an upper (1.4) and lower (0.25) threshold applied.

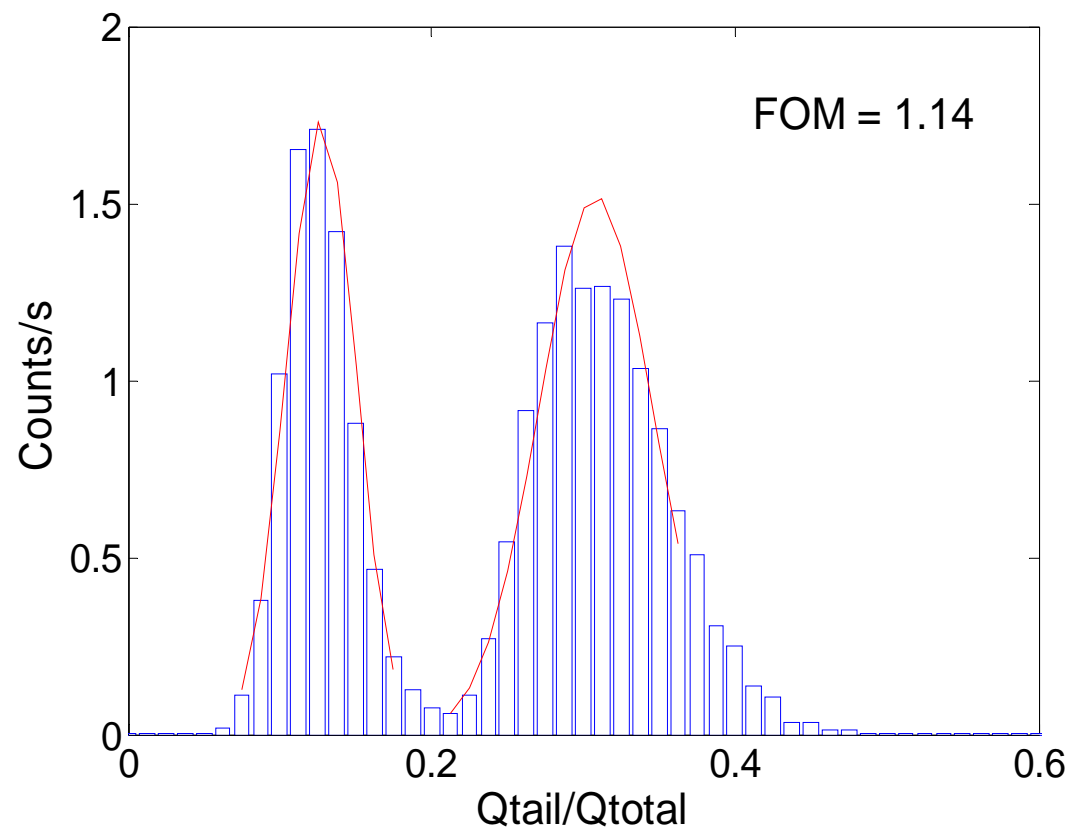

Figure 23: Histogram of the data shown in Figure 22.

The FOM calculated from the data acquired with an AmBe source shielded by $5.08 \mathrm{~cm}$ of lead and Sample 1 was 1.14 . 


\subsection{Additional Results Sample 2}

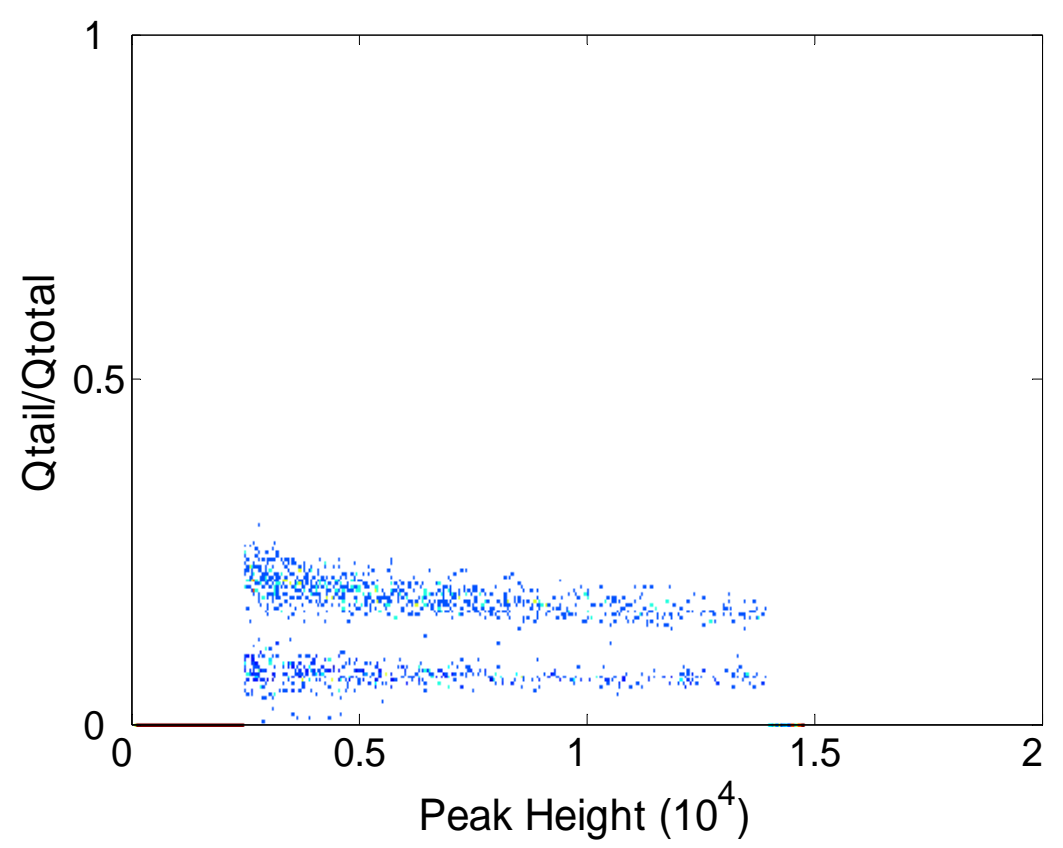

Figure 24: Results from the pulses recorded by Sample 2 in response to a bare AmBe source shielded by 5.08 $\mathrm{cm}$ of $\mathrm{Pb}$ with the pulse peaks aligned and an upper (1.4) and lower (0.25) threshold applied.

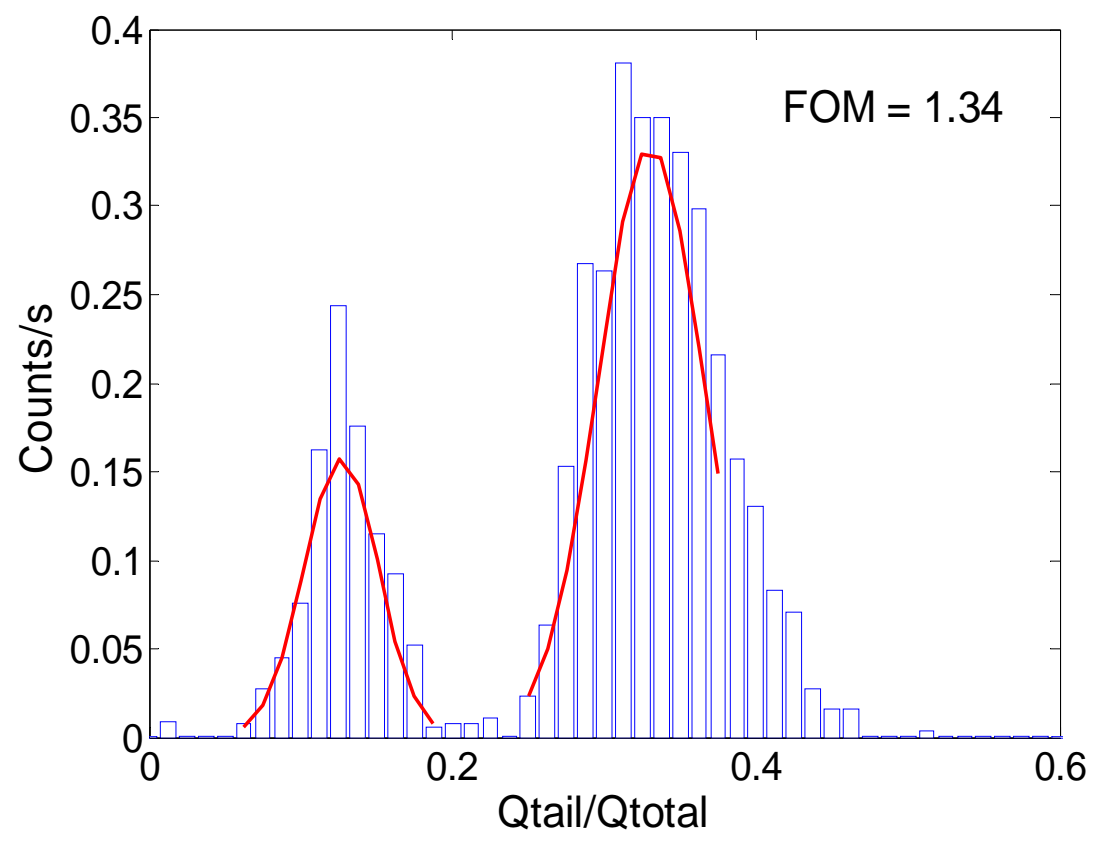

Figure 25: Histogram of the data shown in Figure 24.

The FOM for the gamma ray - neutron separation with an un-moderated AmBe source shielded by $\mathrm{Pb}$ was 1.34 for Sample 2. The Pixie-500 setting B was used which accounts for the improved performance obtained with Sample 2 while the Pixie-500 setting A was used for the AmBe data acquisition with Sample 1. 


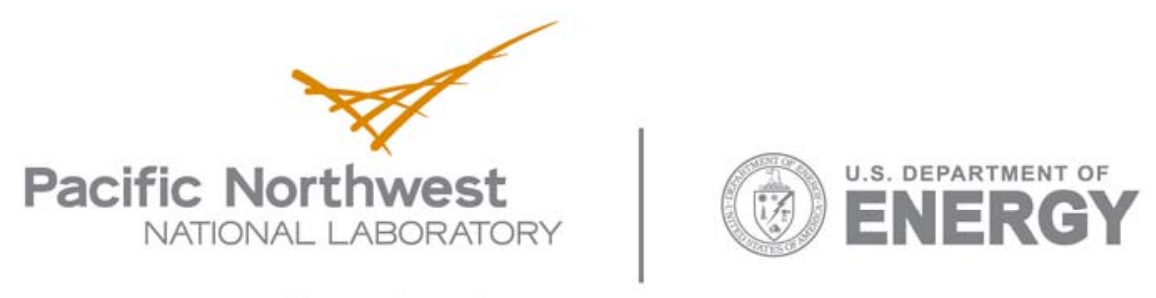

902 Battelle Boulevard

P.O. Box 999

Richland, WA 99352

1-888-375-PNNL (7665)

www.pnl.gov 